\title{
Maximum Planar Subgraphs and Nice Embeddings: Practical Layout Tools
}

\author{
Michael Jünger ${ }^{*}$ and Petra Mutzel ${ }^{* *}$ \\ * Institut für Informatik, Universität zu Köln, Pohligstraße 1, D-50969 Köln, Germany \\ mjuenger@informatik.uni-koeln.de \\ ** Max-Planck-Institut für Informatik, Im Stadtwald, D-66123 Saarbrücken, Germany \\ mutzel@mpi-sb.mpg.de
}

In automatic graph drawing a given graph has to be layed-out in the plane, usually according to a number of topological and aesthetic constraints. Nice drawings for sparse nonplanar graphs can be achieved by determining a maximum planar subgraph and augmenting an embedding of this graph. This approach appears to be of limited value in practice, because the maximum planar subgraph problem is NP-hard.

We attack the maximum planar subgraph problem with a branch and cut technique which gives us quite good and in many cases provably optimum solutions for sparse graphs and very dense graphs. In the theoretical part of the paper, the polytope of all planar subgraphs of a graph $G$ is defined and studied. All subgraphs of a graph $G$, which are subdivisions of $K_{5}$ or $K_{3,3}$, turn out to define facets of this polytope. For cliques contained in $G$, the Euler inequalities turn out to be facetdefining for the planar subgraph polytope. Moreover we introduce the subdivision inequalities, $V_{2 k}$ inequalities and the flower inequalities all of which are facet-defining for the polytope. Furthermore, the composition of inequalities by 2 -sums is investigated.

We also present computational experience with a branch and cut algorithm for the above problem. Our approach is based on an algorithm which searches for forbidden substructures in a graph that contains a subdivision of $K_{5}$ or $K_{3,3}$. These structures give us inequalities which are used as cutting planes.

Finally, we try to convince the reader that the computation of maximum planar subgraphs is indeed a practical tool for finding nice embeddings by applying this method to graphs taken from the literature.

Key words: Maximum planar subgraph, planar subgraph polytope, facets, branch and cut

\section{Introduction}

A graph $G=(V, E)$ is said to be planar, if it can be drawn on the plane such that no two edges intersect geometrically except at a vertex at which they are both incident. According to Kuratowski's Theorem, planar graphs are exactly the graphs that contain no subdivisions of $K_{5}$ or $K_{3,3}$. Given a nonplanar weighted graph with edge weights $w_{e}$ for $e \in E$, we want to delete a set of edges $F$ to obtain a planar subgraph $G^{\prime}=(V, E \backslash F)$ such that the sum of all edge weights $\sum_{e \in E \backslash F} w_{e}$ of $G^{\prime}$ is maximum. In the unweighted case, where $w_{e}=1$ for all edges $e \in E$, the problem consists of finding the minimum number of edges whose deletion from a nonplanar graph gives a planar subgraph.

In either case the problem is NP-hard [GJ79]. The problem can be solved in polynomial time if $G$ is already planar, since planarity testing can be done in linear time [HT74]. If $G=K_{n}$, the 
complete graph on $n$ nodes, or $G=K_{m, n}$, the complete bipartite graph on $n+m$ nodes, it is easy to construct a solution which contains $3 n-6$, resp. $2 n-4$ edges. Since Euler showed that the number of edges in a planar graph on $n$ nodes cannot exceed $3 n-6$, resp. $2 \mathrm{n}-4$, we have solved the unweighted problem in linear time.

A related problem to the unweighted maximum planar subgraph problem is the maximal planar subgraph problem. It consists of finding a planar subgraph $G^{\prime}=\left(V^{\prime}, E^{\prime}\right)$ such that for all edges $e \in E \backslash E^{\prime}$ the addition of $e$ to $G^{\prime}$ destroys the planarity of $G^{\prime}$. Recently Cai, Han and Tarjan [CHT91] described an $\mathrm{O}(|E| \log |V|)$ maximal planarization algorithm based on the Hopcroft-Tarjan planarity testing algorithm, and Kant [K92] generalized the maximal planarization algorithm of Jayakumar et al. [JTS89] for a special class of graphs to an $\mathrm{O}\left(|V|^{2}\right)$ planarization algorithm based on $P Q$-trees of Booth and Lueker [BL76].

For the weighted maximum planar subgraph problem Foulds et al. described some heuristics which deal with complete graphs [FR78, EFG82]. They applied their heuristics to determine good layouts of manufacturing facilities, whose modelling yields very dense graphs. Branch and bound algorithms have been proposed, but they only have a chance on small dense graphs [FR76]. Recently, Goldschmidt and Takvorian presented a two-phase heuristic for solving the unweighted maximum planar subgraph problem [GT92]. They also tried to find a triangulated planar subgraph, but if the density of the graph is not high enough, the heuristic fails.

In automatic graph drawing a given graph has to be layed-out in the plane, usually according to a number of topological and aesthetic constraints. In [TBB88] Tamassia et al. describe their graphtheoretic approach. In the initial phase of the process, an unweighted maximum planar subgraph of the input graph is determined, which is then used as the basis for the layout of the original graph. The graphs occuring in such applications are relatively sparse, so that the above heuristic fails.

We attack the problem with a branch and cut technique. This approach gives us quite good and in many cases provably optimal solutions for sparse graphs and very dense graphs.

In section 2, we define the planar subgraph polytope $\mathcal{P} \mathcal{L} \mathcal{S}(G)$. Some basic facts about this polytope are given. Among others all the minimal nonplanar subgraphs of a graph $G$, which are exactly the subdivisions of $K_{5}$ and $K_{3,3}$ contained in $G$, turn out to define facets of $\mathcal{P} \mathcal{L S}(G)$. Moreover, the "Euler inequalities", which state that a planar (bipartite) graph on $n$ nodes has at most $3 n-6$ $(2 n-4)$ edges, are shown to be facet-defining for the polytope. Furthermore subdivision inequalities, $V_{2 k}$ inequalities and flower inequalities are introduced all of which are shown to be facet-defining for the polytope. In addition, some operations like lifting, edge splitting, edge contraction and composition of facet-defining inequalities by 2 -sums are examined. In section 3 , we present the algorithm. The computational results are given in section 4 .

\section{The Planar Subgraph Polytope}

The theoretical background of our method is based on polyhedral combinatorics, a subfield of combinatorial optimization which aims at describing combinatorial optimization problems as linear programs and solving these with special purpose methods. We outline the approach for the maximum planar subgraph problem for general graphs. For a graph $G$ we denote its node set by $V(G)$ and its edge set by $E(G)$. Edges of $G$ are denoted by their endnodes, i. e. we write $e=(v, w)$ or $e=(w, v)$ for $e \in E(G)$. We say that $G^{\prime}=(W, F)$ is a subgraph of $G=(V, E)$ if $W \subseteq V$ and $F \subseteq E$. If $G^{\prime}$ contains all the edges of $G$ that join two vertices in $W$ then $G^{\prime}$ is said to be induced by $W$. If $W$ consists of exactly the vertices on which edges in $F$ are incident, then $G^{\prime}$ is said to be induced by $F$ and we write $G^{\prime}=G[F]$. If $W \subseteq V(G)$, we define $E[W]:=\{(v, w) \in E \mid v, w \in W\}$. The degree of a vertex $v$ of $V(G)$ is the number of edges incident to $v$, and is written as $\operatorname{deg}(v)$. Furthermore, for $F \subseteq E(G)$ we use the notation $x(F):=\sum_{e \in F} x_{e}$. 


\subsection{Polyhedral Combinatorics}

A polytope in $\mathbf{R}^{n}$ is the convex hull of finitely many points, or equivalently, a polytope is a bounded subset of $\mathbf{R}^{n}$ that is the intersection of finitely many halfspaces. Those points of a polytope $P$ which are not representable as a convex combination of other points in $P$ are the vertices of $P$.

The dimension of a polytope in $\mathbf{R}^{n}$ is the maximum number of affinely independent points in $P$ minus 1. $P$ is full-dimensional if its dimension is $n$. An inequality $c^{T} x \leq c_{0}$ is valid for $P \subseteq \mathbf{R}^{n}$ if $P \subseteq\left\{x \in \mathbf{R}^{n} \mid c^{T} x \leq c_{0}\right\}$. If $c^{T} x \leq c_{0}$ is valid then $F:=\left\{x \in P \mid c^{T} x=c_{0}\right\}$ is a face of $P$. A facet is a face of dimension one less than the dimension of $P$. An important theorem of polyhedral theory states that for a full-dimensional polytope every facet is defined by a unique (up to multiplication by a positive constant) inequality (i. e., if $F=\left\{x \in P \mid c^{T} x=c_{0}\right\}=\left\{x \in P \mid a^{T} x=a_{0}\right\}$ is a facet of $P$ and $c^{T} x \leq c_{0}$ and $a^{T} x \leq a_{0}$ are valid for $P$ then $c=\lambda a, c_{0}=\lambda a_{0}$ for some $\lambda>0$ ), and moreover, that every system of inequalities describing $P$ completely must contain, for each facet $F$ of $P$, at least one inequality defining $F$. This shows that in order to describe $P$ in the form $P=\{x \mid A x \leq b\}$ one has to know the inequalities defining facets of $P$.

Let us now turn to the maximum planar subgraph problem. Suppose a graph $G=(V, E)$ with edge weights $w_{e}$ for all $e \in E$ is given. Let $\mathcal{P}_{\mathcal{G}}$ be the set of all planar edge-induced subgraphs of $G$. For each planar subgraph $P=G[F] \in \mathcal{P}_{\mathcal{G}}$, we define its incidence vector $\chi^{P} \in \mathbf{R}^{E}$ by setting $\chi_{e}^{P}=1$ if $e \in F$ and $\chi_{e}^{P}=0$ if $e \notin F$. This yields a 1-1-correspondence of the planar subgraphs with certain $\{0,1\}$-vectors in $\mathbf{R}^{E}$. The planar subgraph polytope $\mathcal{P L S}(G)$ of $G$ is defined as the convex hull over all incidence vectors of planar subgraphs of $G$

$$
\mathcal{P} \mathcal{L} S(G):=\operatorname{conv}\left\{\chi^{P} \in \mathbf{R}^{E} \mid P \in \mathcal{P}_{G}\right\}
$$

The problem of finding a planar subgraph $P$ of $G$ with weight $w(P)$ as large as possible can be written as the linear program

$$
\max \left\{w^{T} x \mid x \in \mathcal{P} \mathcal{L} \mathcal{S}(G)\right\},
$$

since the vertices of the polytope $\mathcal{P} \mathcal{L S}(G)$ are exactly the incidence vectors of the planar subgraphs of $G$. In order to apply linear programming techniques to solve this LP one has to represent $\mathcal{P L S}(G)$ as the solution of an inequality system. Due to the NP-hardness of our problem, we cannot expect to be able to find a full description of $\mathcal{P} \mathcal{L S}(G)$ by linear inequalities. Nevertheless, a partial description of the facial structure of $\mathcal{P} \mathcal{L S}(G)$ by linear inequalities is useful for the design of a "branch and cut"-algorithm, because such a description defines a relaxation of the original problem. Such relaxations can be solved within a branch and bound framework via cutting plane techniques and linear programming in order to produce tight bounds. In an irredundant description of $\mathcal{P} \mathcal{L S}(G)$ by linear inequalities only facet-defining inequalities are present. For efficiency, also in a partial description by inequalities, we concentrate on those valid inequalities for $\mathcal{P} \mathcal{L} \mathcal{S}(G)$ which are facet-defining. An excellent introduction into the theory of polyhedral combinatorics is given by Pulleyblank in [P89].

In the following we give a partial description of the facial structure of $\mathcal{P} \mathcal{L} \mathcal{S}(G)$. The set of all planar subgraphs $\mathcal{P}_{G}$ of $G$ is an independence system, since every subgraph of a planar graph is planar.

Lemma 1 The dimension of the $\mathcal{P} \mathcal{L S}$-polytope of $G=(V, E)$ is $|E|$, so it is full dimensional. For all edges $e \in E$ the inequalities $x_{e} \geq 0$ and $x_{e} \leq 1$ define facets of $\mathcal{P} \mathcal{L S}(G)$.

Proof. The first part follows directly from the properties of independence systems. It is also easy to see that the inequality $x_{e} \leq 1$ is facet-defining by directly choosing the $|E|-1$ edge sets $F_{i}=\left\{e \cup e_{i}\right\}$ for all $e_{i} \in E \backslash\{e\}$. Together with $F=\{e\}$ their incidence vectors give linear independent incidence vectors of planar subgraphs, which satisfy the inequality $x_{e} \leq 1$ with equality. 


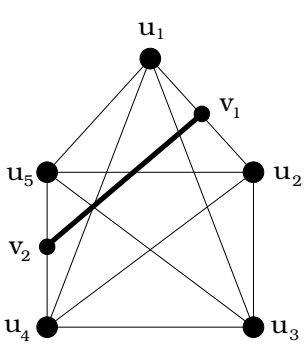

(a)

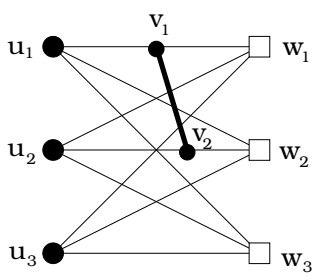

(e)

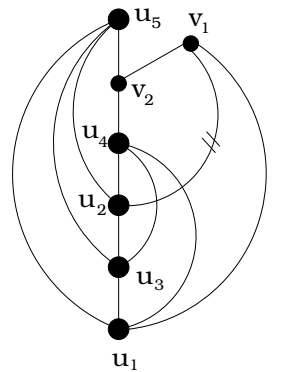

(b)

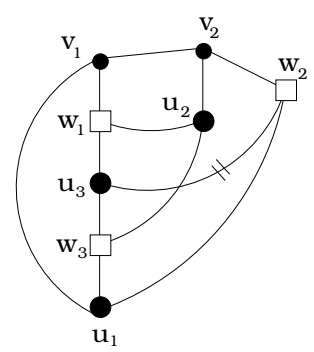

(f)

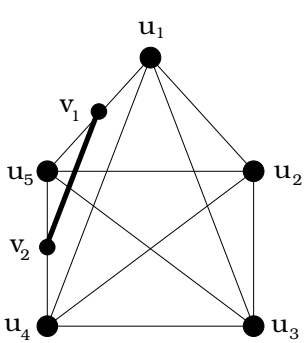

(c)

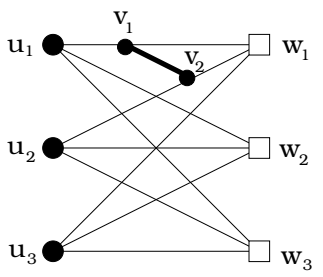

(g)

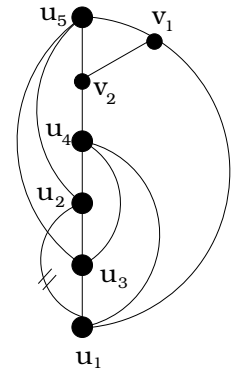

(d)

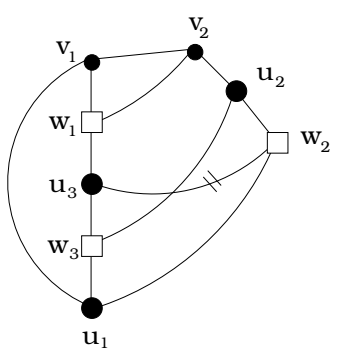

(h)

Figure 1

\subsection{Kuratowski and Euler inequalities}

A minimal nonplanar graph is a nonplanar graph for which the removal of an arbitrary edge yields a planar graph. Minimal nonplanar graphs are the circuits in the independence system $\mathcal{P}_{G}$. Since by Kuratowski's Theorem every nonplanar graph contains a subdivision of $K_{5}$ or $K_{3,3}$, one can easily observe that the minimal nonplanar graphs are exactly the subdivisions of $K_{5}$ or $K_{3,3}$. In the following we will examine their properties. The following lemma is obvious.

Lemma 2 For a minimal nonplanar subgraph $G[F]$ of a nonplanar graph $G=(V, E), F \subseteq E$, an arbitrary edge $f \in F$ and an edge $e \in E \backslash F$ with one endnode not in $V(F)$ the graph $G\left[F^{\prime}\right]$ induced by $F^{\prime}=F \backslash\{f\} \cup\{e\}$ is planar.

Theorem 1 For a minimal nonplanar subgraph $G[F]$ of a nonplanar graph $G=(V, E)$ and an edge $e \in E \backslash F$ there exists an edge $f \in F$ such that the graph $G\left[F^{\prime}\right]$ induced by $F^{\prime}=F \backslash\{f\} \cup\{e\}$ is planar.

Proof. Consider first a minimal nonplanar subgraph which is a subdivision of $K_{5}$. We denote the nodes which are no subdivision nodes by $u_{1}, \ldots, u_{5}$. Let us assume that we add the edge $\left(v_{1}, v_{2}\right)$ with endnodes $v_{1}$ on the path from $u_{1}$ to $u_{2}$ and $v_{2}$ on the path from $u_{4}$ to $u_{5}$ (see Figure 1(a)). An embedding of this graph is given in Figure 1(b). Clearly, removing one edge on the path from $u_{2}$ to $v_{1}$ will lead to a planar graph. If the edge $\left(v_{1}, v_{2}\right)$ joins two adjacent paths, for example $v_{1}$ lies now on the path from $u_{1}$ to $u_{5}$ (see Figure 1(c)), then again the deletion of one edge on the path from $u_{1}$ to $u_{2}$ gives a planar graph (see Figure 1(d)). The case in which not both nodes $v_{1}$ and $v_{2}$ are subdivision nodes can be obtained from the above via a suitable contraction. All other cases are symmetric to the above.

Now consider a graph which is a subdivision of $K_{3,3}$. Again denote the nodes which are no subdivision nodes by $u_{1}, u_{2}, u_{3}, w_{1}, w_{2}, w_{3}$. Let us assume that we add the edge $\left(v_{1}, v_{2}\right)$ with endnodes $v_{1}$ on the path from $u_{1}$ to $w_{1}$ and $v_{1}$ on the path from $u_{2}$ to $w_{2}$ (see Figure 1(e)). By considering the embedding of the graph given in Figure 1(f) it is obvious that the graph will be planar, if one edge on the path from $w_{2}$ to $u_{3}$ is deleted. The case in which the edge $\left(v_{1}, v_{2}\right)$ joins two adjacent paths is treated in Figure 1(g)-(h). 
This theorem leads to an important theoretical result of this paper.

Theorem 2 (Kuratowski inequalities) For all minimal nonplanar subgraphs $G^{\prime}=\left(V^{\prime}, F\right)$ of $G=(V, E)$ the Kuratowski inequality $x(F) \leq|F|-1$ defines a facet of $\mathcal{P} \mathcal{L S}(G)$.

Proof. Take the $|F|$ edge sets $F \backslash\{f\}$ for all edges $f \in F$. Consider an edge $e \in E \backslash F$, add it to $F$ and delete an edge $f \in F$ such that the subgraph induced by $F^{\prime}=F \backslash\{f\} \cup\{e\}$ is planar, which is possible due to Theorem 1. Do this for all edges $e \in E \backslash F$. All of the $|E|$ edge sets satisfy the inequality $x(F) \leq|F|-1$ at equality and the graphs induced by them are planar. Consider the corresponding matrix $A$ whose rows are the incidence vectors of these edge sets. If in the rows of $A$ the edges in $F$ appear before the remaining ones, it is obvious that $A$ has rank $|E|$. Thus these $|E|$ planar graphs are all linearly independent and the theorem follows.

Corollary 1 Let $K_{5}\left(\operatorname{resp} . K_{3,3}\right)$ be contained in $G=(V, E)$. Then $x\left(K_{5}\right) \leq 9\left(\operatorname{resp} . x\left(K_{3,3}\right) \leq 8\right)$ defines a facet of $\mathcal{P} \mathcal{L S}(G)$.

The facet-defining property of $K_{5}$ and $K_{3,3}$ is not very astonishing. Another class of inequalities, which plays an important role in the theory of planar graphs, can be obtained from Euler's formula for the relationships of vertices, edges and faces in a plane connected graph.

Lemma 3 (Euler inequalities) For $G=(V, E)$ and $V^{\prime} \subseteq V$ let $E^{\prime}:=E\left[V^{\prime}\right]$ and $G^{\prime}=\left(V^{\prime}, E^{\prime}\right)$ the inequality $x\left(E^{\prime}\right) \leq 3\left|V^{\prime}\right|-6$ is valid for $\mathcal{P} \mathcal{L} \mathcal{S}(G)$. If $G^{\prime}$ is bipartite, the inequality intensifies to $x\left(E^{\prime}\right) \leq 2\left|V^{\prime}\right|-4$.

Moreover, if the graph $G=(V, E)$ is dense, the above inequality may yield a facet, like it is the case for $G=K_{n}$, resp. $G=K_{m, n}$.

Theorem 3 For the complete graph on $n$ nodes $G=K_{n}=(V, E)$ the inequality $x(E) \leq 3|V|-6$ defines a facet for $\mathcal{P} \mathcal{L S}(G)$ for $n \geq 5$. If $G$ is the complete bipartite graph $K_{m, n}$, then the inequality $x(E) \leq 2|V|-4$ defines a facet for $\mathcal{P} \mathcal{L S}(G)$ for $m, n \geq 3$.

Proof. We show the theorem for the complete bipartite graph $K_{m, n}$. The proof for complete graphs is similar, but not as complicated and much shorter. Let us assume that $G=(U, W, E)$ with $|U|=m \geq n=|W| \geq 3$. For notational convenience we denote $x(E) \leq 2(|U|+|W|)-4$ by $a^{T} x \leq a_{0}$. Suppose now that $c^{T} x \leq c_{0}$ is a valid inequality for $\mathcal{P} \mathcal{L S}(G)$ satisfying $\{x \in \mathcal{P} \mathcal{L S}(G) \mid$ $\left.a^{T} x=a_{0}\right\} \subseteq\left\{x \in \mathcal{P} \mathcal{L S}(G) \mid c^{T} x=c_{0}\right\}$. We show that for some $\alpha \geq 0$ we have $c^{T}=\alpha a^{T}$ and $c_{0}=\alpha a_{0}$. Consider the plane graph $P$ shown in Figure 2(a), where $U=\left\{u_{1}, \ldots, u_{m}\right\}$ and $W=\left\{w_{1}, \ldots, w_{n}\right\}$. $P$ has exactly $(3 n-2)+(n-2)+2(m-n)=2(m+n)-4$ edges, thus its incidence vector $\chi^{P}$ satisfies $a^{T} \chi^{P}=a_{0}$ and hence also $c^{T} \chi^{P}=c_{0}$.

Let us construct a new graph $P_{2}^{i}$ by deleting the edge $\left(w_{i+1}, u_{i+1}\right)$ and adding the edge $\left(w_{i}, u_{i+2}\right)$. $P_{2}^{i}$ is still planar and satisfies $a^{T} \chi^{P_{2}^{i}}=a_{0}$ and hence $c^{T} \chi^{P_{2}^{i}}=c_{0}$. This implies $0=c_{0}-c_{0}=c^{T} \chi^{P}-$ $c^{T} \chi^{P_{2}^{i}}=c_{w_{i+1} u_{i+1}}-c_{w_{i} u_{i+2}}$, thus (1) $c_{w_{2} u_{2}}=c_{w_{1} u_{3}}$. In general, we construct $P_{h}^{i}$ from $P$ by adding the edges $\left(w_{i}, u_{i+2}\right), \ldots,\left(w_{i}, u_{i+h}\right)$ and deleting the edges $\left(w_{i+1}, u_{i+1}\right), \ldots,\left(w_{i+h-1}, u_{i+h-1}\right)$ for $i=$ $1, \ldots, n-2$ and $h=2, \ldots, n-i$. The graphs $P_{h}^{i}$ are obviously still planar and satisfy $c^{T} \chi^{P_{h}^{i}}=c_{0}$ with equality (Figure 2(b) shows $\left.P_{3}^{1}\right)$. Subtraction yields $c^{T} \chi^{P_{h-1}^{i}}-c^{T} \chi^{P_{h}^{i}}=\left(\left(c_{w_{i} u_{i+2}}+\cdots+c_{w_{i} u_{i+h-1}}\right)-\right.$ $\left.\left(c_{w_{i+1} u_{i+1}}+\cdots+c_{w_{i+h-2} u_{i+h-2}}\right)\right)-\left(\left(c_{w_{i} u_{i+2}}+\cdots+c_{w_{i} u_{i+h}}\right)-\left(c_{w_{i+1} u_{i+1}}+\cdots+c_{w_{i+h-1} u_{i+h-1}}\right)\right)=$ $-c_{w_{i} u_{i+h}}+c_{w_{i+h-1} u_{i+h-1}}$. Together with (1) we have (2) $c_{w_{i} u_{i+h}}=c_{w_{i+h-1} u_{i+h-1}}$ for $i=1, \ldots, n-2$ and $h=2, \ldots, n-i$. Symmetrically, we get the same for $u_{i}$, that is $c_{u_{i} w_{i+h}}=c_{w_{i+h-1} u_{i+h-1}}$ for $i=2, \ldots, n-2$ and $h=2, \ldots, n-i$.

Next let us construct $F_{k}$ similar as $P_{h}^{i}$ from $P$ by adding the edges $\left(w_{n-2}, u_{n}\right), \ldots,\left(w_{n-k}, u_{n}\right)$ and deleting the edges $\left(u_{n-1}, w_{n-1}\right), \ldots,\left(u_{n-k+1}, w_{n-k+1}\right)$ for $k=2, \ldots, n-1$. Subtraction of $c\left(F_{k-1}\right)$ from $c\left(F_{k}\right)$ yields $c_{w_{n-k} u_{n}}=c_{u_{n-k+1} w_{n-k+1}}$ for $k=3, \ldots, n-1$. Together with (2) we 


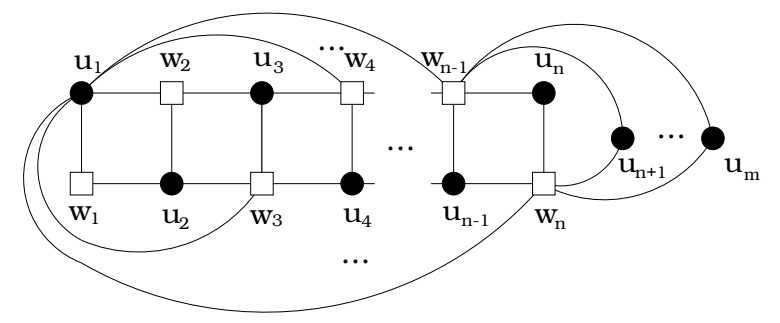

(a)

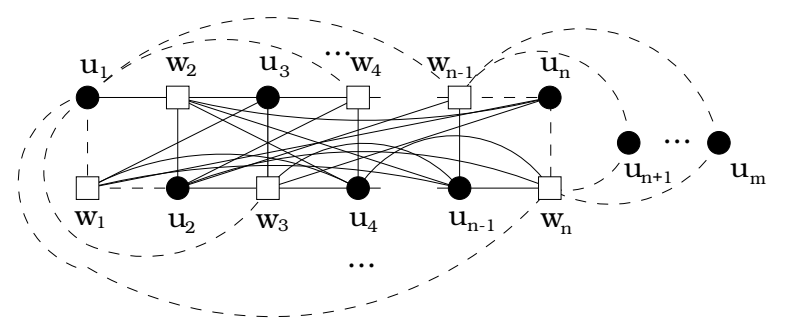

(c)

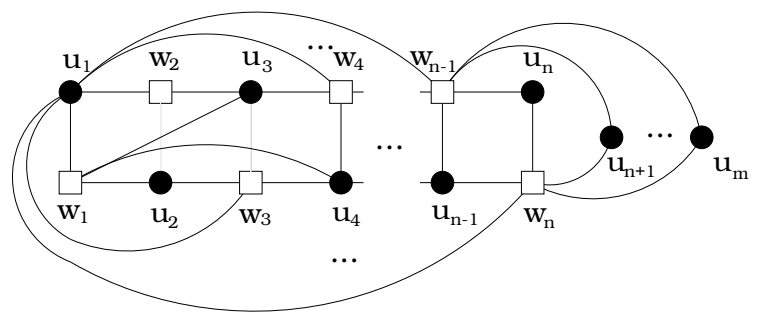

(b)

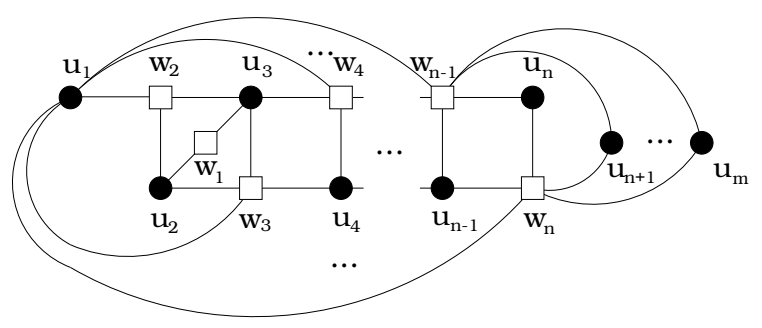

(d)

Figure 2

have $c_{w_{n-1} u_{n-1}}=c_{w_{n-h} u_{n}}=c_{u_{n-h+1} w_{n-h+1}}=c_{w_{(n-1)-(h-2)} u_{(n-1)-(h-2)}}=c_{w_{n-1-j} u_{n-1-j}}$ for $j=$ $1, \ldots, n-3$.

The planarity of the graph $H_{i}^{w}$ arising from $P$ by adding the edge $\left(w_{i}, u_{i+2}\right)$ and deleting edge $\left(u_{i+1}, w_{i+2}\right)$ for $i=1, \ldots, n-2$ is evident by Figure $2(\mathrm{a})$. The same holds for $H_{i}^{u}$ arising from $P$ by adding the edge $\left(u_{i}, w_{i+2}\right)$ and deleting $\left(w_{i}, u_{i+1}\right)$ for $i=2, \ldots, n-2$. We also preserve planarity by adding $\left(w_{1}, u_{3}\right)$ and deleting $\left(u_{1}, w_{2}\right)$ (If $n=3$ the nodes $u_{n+h}$ for $h=1, \ldots, m-n$ have to be embedded into a different face). This way we get the $c$-values for the deleted edges.

So far we have shown equality of all coefficients $c_{e}$ of edges $e_{i}^{h}=\left(u_{i}, w_{i+h}\right)$ for $i=2, \ldots, n-1$ and $h=0, \ldots, n-i$, the edges $f_{i}^{h}=\left(w_{i}, u_{i+h}\right)$ for $i=1, \ldots, n-2$ and $h=0, \ldots, n-i$, where $i+h>2$, and $e=\left(u_{1}, w_{2}\right)$ (see Figure 2(c), the values of the solid drawn edges are known). Equality of the $c$-values for the edges $e_{1}^{j}=\left(u_{1}, w_{j}\right)$ for $j=3, \ldots, n-2$ is obtained by replacing $e_{1}^{j}$ with $\left(w_{j-2}, u_{j+1}\right)$, which keeps planarity. The values of $\left(u_{1}, w_{n-1}\right)$ and $\left(u_{1}, w_{n}\right)$ are obtained by replacing them with $\left(w_{n-2}, u_{m}\right)$ and $\left(w_{n-3}, u_{m}\right)$, respectively.

For the case $m=n$ we have shown equality of the coefficients of almost all edges but $\left(u_{1}, w_{1}\right)$, $\left(w_{1}, u_{2}\right),\left(w_{n-1}, u_{n}\right)$ and $\left(u_{n}, w_{n}\right)$. By interchanging node $u_{2}$ with $u_{3}$ in $P$ (see Figure 2(a)) we obtain again a planar graph with the same weight and get $c_{w_{1} u_{2}}+c_{u_{3} w_{4}}=c_{w_{1} u_{3}}+c_{u_{2} w_{4}}$, where all values except $c_{w_{1} u_{2}}$ are known to be equal. The $c$-values of the remaining edges can be obtained by the following construction. Delete edge $\left(u_{1}, w_{1}\right)$ from $P$ and add $\left(w_{1}, u_{3}\right)$. We observe that this graph is still planar (see Figure 2(d)) and satisfies $a^{T} x=a_{0}$ with equality, hence $c^{T} x=c_{0}$, which implies $c_{u_{1} w_{1}}=c_{w_{1} u_{3}}$. By the same construction in the rightmost rectangle we get $c_{u_{n} w_{n}}=c_{w_{n-2} u_{n}}$. In order to get $c_{w_{n-1} u_{n}}=c_{w_{n-2} u_{n}}$, we delete the edge $\left(w_{n-1}, u_{n}\right)$ in $P$ (see Figure 2(a)) and embed $u_{n}$ into the face determined by $\left\{u_{1}, w_{n-2}, u_{n-1}, w_{n}\right\}$. Obviously, the graph obtained by adding the edge $\left(w_{n-2}, u_{n}\right)$ is planar.

In case $m>n$ we need to show the equality of the $c$-values for all edges $\left(u_{n+h}, w_{i}\right)$ for $h=$ $1, \ldots, m-n$ and $i=1, \ldots, n$. We embed the node $u_{n+h}$ (for fixed $h$ ) into the first rectangle determined by $\left\{u_{1}, w_{2}, u_{2}, w_{1}\right\}$. For this, we have to delete the edges $\left(u_{n+h}, w_{n-1}\right)$ and $\left(u_{n+h}, w_{n}\right)$ from $P$ and to add $\left(w_{1}, u_{n+h}\right)$ and $\left(w_{2}, u_{n+h}\right)$. Let $R^{i}$ denote the graphs obtained by doing this for each of the rectangles $\left\{u_{i}, w_{i+1}, u_{i+1}, w_{i}\right\}$ for $i=1, \ldots, n-1$. By subtraction of $c\left(R^{i-1}\right)$ from $c\left(R^{i}\right)$ we get the equations $c_{w_{1} u_{n+h}}=c_{w_{3} u_{n+h}}=\cdots=c_{w_{n} u_{n+h}}$ and $c_{w_{2} u_{n+h}}=c_{w_{4} u_{n+h}}=\cdots=c_{w_{n-1} u_{n+h}}$ 
if $n$ is odd. Let $R^{\prime}$ be the graph obtained from $R^{1}$ by deleting edge $\left(u_{2}, w_{2}\right)$ and adding edge $\left(u_{n+h}, w_{3}\right)$. By subtraction of $c\left(R^{\prime}\right)$ from $c\left(R^{1}\right)$ we get $c_{w_{3} u_{n+h}}=c_{u_{2} w_{2}}$. From $R^{2}$ we get by the same construction $c_{w_{4} u_{n+h}}=c_{u_{3} w_{3}}$ and so we have shown the equality of the $c$-values for all edges in $G=K_{m, n}$. Thus setting $\alpha=c_{0} / a_{0}$ proves the theorem.

The facet-defining property also holds if we delete one arbitrary edge of $K_{n}$, which is not the case for $K_{m, n}$. For the graph $K_{3,4}$ with one deleted edge the inequality just yields a face of dimension $|E|-2$. The proof of Corollary 2 is essentially the same as for $K_{n}$.

Corollary 2 For the complete graph $G=(V, E)$ on $n \geq 6$ nodes where one arbitrary edge $e$ is removed, the inequality $x(E) \leq 3|V|-6$ is facet-defining for $\mathcal{P} \mathcal{L S}(G)$.

One may think that the same must also hold for complete graphs in which two edges are removed. But this is not the case. For the graph $K_{6}$ the above inequality is not facet-defining for any pair of deleted edges.

The inequalities considered up to this point have all coefficients $c_{e}=1$. This is no longer true for the following inequalities.

\subsection{Subdivision, $\mathrm{V}_{2 \mathrm{k}}$ and flower inequalities}

Theorem 4 Let $G=(V, E)$ be a subdivision of $K_{5}$ on the nodes $u_{1}, \ldots, u_{5}, v_{1}, v_{2}$ extended by the edge $\left(v_{1}, v_{2}\right)$, where $v_{1}$ and $v_{2}$ denote the subdivision nodes. Furthermore assume $\left(u_{i}, v_{1}\right)$, $\left(u_{j}, v_{1}\right) \in E$ and $\left(u_{k}, v_{2}\right),\left(u_{l}, v_{2}\right) \in E$ with $i \neq j, k \neq l, j \neq k$ and $j \neq l$.

If $i=k$, we define $U=\left\{\left(u_{h}, u_{i}\right),\left(u_{h}, u_{j}\right),\left(u_{h}, u_{l}\right)\right.$ for $\left.h \neq i, j, l\right\} \cup\left\{\left(u_{j}, v_{1}\right),\left(u_{l}, v_{2}\right)\right\}$. The definition of $U$ for the case $i=l$ is symmetric. If $i, j, k$ and $l$ are pairwise distinct, we define $U=\left\{\left(u_{i}, v_{1}\right),\left(u_{j}, v_{1}\right),\left(u_{k}, v_{2}\right),\left(u_{l}, v_{2}\right)\right\}$.

Let $c_{e}=2$ for all edges $e \in U$ and $c_{e}=1$ for the remaining edges $e \notin U$. Then the inequality $2 x(U)+x(E \backslash U) \leq c(E)-2$ is facet-defining for $\mathcal{P} \mathcal{L S}(G)$.

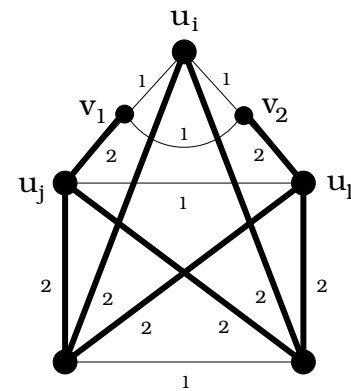

(a)

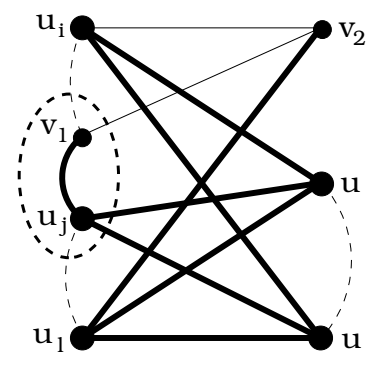

(b)

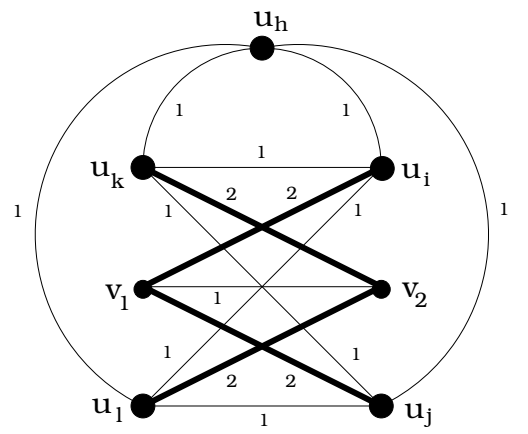

(c)

Figure 3

Proof. First consider the case in which $i=k$ (see Figure 3(a)). Each subdivision of $K_{3,3}$ contained in $G$ must exactly have one node of $\left\{v_{1}, v_{2}\right\}$ as subdivision node, say $v_{1}$. Such a subdivision must be of the form shown in Figure 3(b). The edge set is $F_{v_{1}}=U \cup\left\{\left(v_{1}, v_{2}\right),\left(u_{i}, v_{2}\right)\right\}$. Symmetrically, if $v_{2}$ is the subdivision node, we obtain the edge set $F_{v_{2}}=U \cup\left\{\left(v_{1}, v_{2}\right),\left(u_{i}, v_{1}\right)\right\}$. Whenever an edge $e \notin U$ is deleted from $G$, either a subdivision of $K_{5}$ is left or one of the above subdivisions of $K_{3,3}$ is still contained in the remaining graph. Thus validity is shown. The common edge set of all the minimal nonplanar graphs is exactly $U$. Thus $G \backslash\{e\}$ for any $e \in U$ is planar. By deleting the edge $\left(v_{1}, v_{2}\right)$ in $G$ we obtain exactly the subdivision of $K_{5}$. Together with $\left(v_{1}, v_{2}\right)$ we can delete any other edge to get a planar graph, in particular we can choose any additional edge 


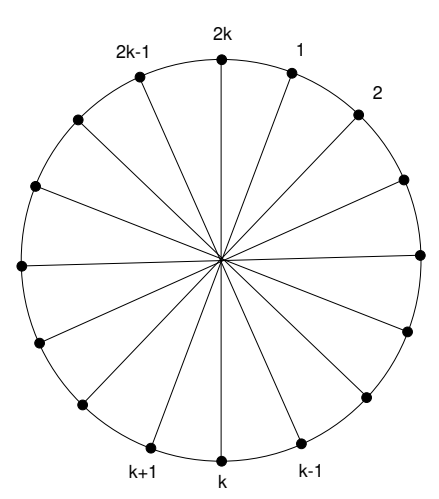

(a) Graph $V_{2 k}$

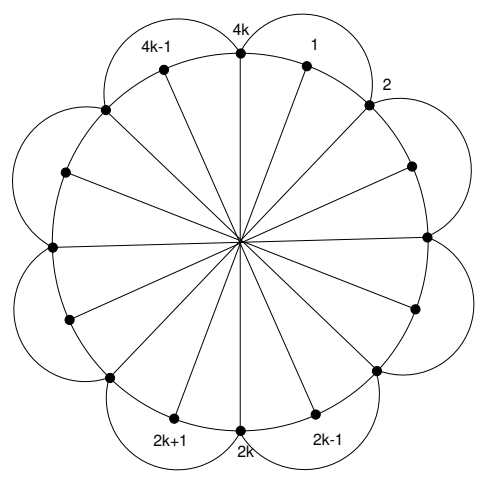

(b) Graph $F_{4 k}$

Figure 4

$e \in E \backslash\left(U \cup\left\{\left(v_{1}, v_{2}\right)\right\}\right)$. It is also obvious that the removal of the edges $\left(u_{i}, v_{1}\right)$ and $\left(u_{i}, v_{2}\right)$ from $G$ results in a planar graph. Thus we have found $|E|$ incidence vectors of planar subgraphs, which all satisfy the inequality $2 x(U)+x(E \backslash U) \leq c(E)-2$ with equality. The linear independence of these vectors can easily be verified.

Let us consider the second case in which $i \neq k, l$ and $j \neq k, l$. An embedding of the graph is shown in Figure 3(c). After the deletion of the edge $\left(u_{i}, u_{k}\right)$ there is still a path $u_{i}, u_{h}, u_{k}$ between $u_{i}$ and $u_{k}$, which preserves the presence of a subdivision of $K_{3,3}$. The same applies for the edges $\left(u_{i}, u_{l}\right)$, $\left(u_{j}, u_{k}\right)$ and $\left(u_{j}, u_{l}\right)$. If $\left(v_{1}, v_{2}\right)$ is deleted, the resulting graph is a subdivision of $K_{5}$. Since the common edge set of all the minimal nonplanar graphs is exactly $U$, we have to delete either one edge in $U$ or at least two of the edges not in $U$ to get a planar graph. Thus validity is shown. Together with the edge $\left(v_{1}, v_{2}\right)$ we can delete any other edge in $E \backslash U$ in order to get a planar graph. It is also obvious that the deletion of the edges $\left(u_{k}, u_{j}\right)$ and $\left(u_{i}, u_{l}\right)$ also yields a planar graph. Again we have $|E|$ incidence vectors of planar graph, which are linearly independent and satisfy the inequality $2 x(U)+x(E \backslash U) \leq c(E)-2$ with equality.

In the following we introduce two new classes of graphs both of which can be shown to be facetdefining for the planar subgraph polytope. For $k \in \mathbf{N}, k \geq 2$, we define the graph $\mathbf{V}_{\mathbf{2 k}}$ via $V\left(V_{2 k}\right)=\{1,2, \ldots, 2 k\}$ and $E\left(V_{2 k}\right)=C_{2 k} \cup D_{2 k}$ with $C_{2 k}=\{(i, i+1) \mid i=1, \ldots, 2 k-1\} \cup\{(2 k, 1)\}$ and $D_{2 k}=\{(i, i+k) \mid i=1,2, \ldots, k\}$, and the flower graph $F_{4 k}$ via $V\left(F_{4 k}\right)=\{1,2, \ldots, 4 k\}$ and $E\left(F_{4 k}\right)=C_{4 k} \cup D_{4 k} \cup B_{4 k}$ with $C_{4 k}=\{(i, i+1) \mid i=1, \ldots, 4 k-1\} \cup\{(4 k, 1)\}, D_{4 k}=\{(i, i+2 k) \mid$ $i=1,2, \ldots, 2 k\}$ and $B_{4 k}=\{(2 i, 2 i+2) \mid i=1,2, \ldots, 2 k-1\} \cup\{(4 k, 2)\}$ (see Figure 4). For $k=3$, the graph $V_{2 k}$ is identical to $K_{3,3}$, giving the corresponding Kuratowski inequality.

Theorem 5 For the graph $V_{2 k}=(V, E)$ with $E=C_{2 k} \cup D_{2 k}$ and $k \geq 3$ the $V_{2 k}$ inequality

$$
(k-2) x\left(C_{2 k}\right)+x\left(D_{2 k}\right) \leq 2(k-1)^{2}
$$

is facet-defining for $\mathcal{P} \mathcal{L S}\left(V_{2 k}\right)$.

Proof. The $V_{2 k}$ inequality is valid, since we have to delete either one edge of $C_{2 k}$ or at least $k-2$ edges of $D_{2 k}$ from $V_{2 k}$ in order to get a planar graph. The latter part can be verified by the observation that the outer cycle $C_{2 k}$ together with any 3 diagonals (edges of $D_{2 k}$ ) represents a subdivision of $K_{3,3}$.

Obviously, $C_{2 k} \cup\left\{d_{1}, d_{2}\right\}$ with any $d_{1}, d_{2} \in D_{2 k}$ is planar. Also the graph $V_{2 k}-\{e\}$ with any $e \in C_{2 k}$ is planar (see Figure 5 ). Take the $2 k$ incidence vectors of the graphs arising from removing one edge of $C_{2 k}$, the $k-1$ incidence vectors of the graphs arising from taking all edges of $C_{2 k}$ together with the edge $d_{1}$ and $d_{i}$ of $D_{2 k}\left(d_{i} \neq d_{1}\right)$ and the incidence vector of the graph arising from taking the 


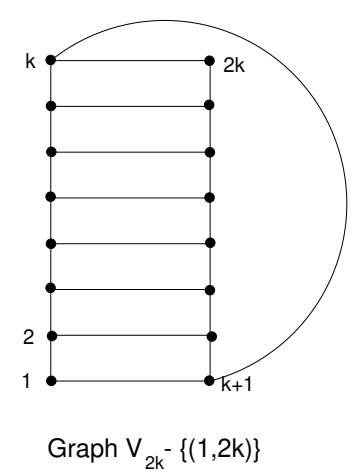

Figure 5

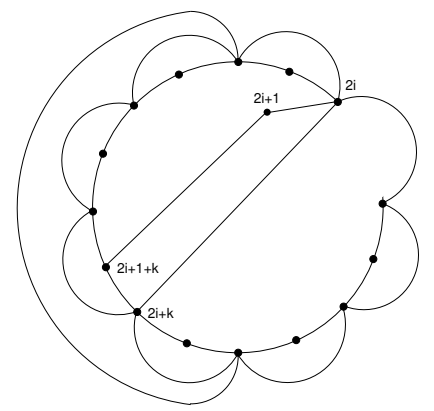

(a) Graph $\mathrm{P}_{2}$

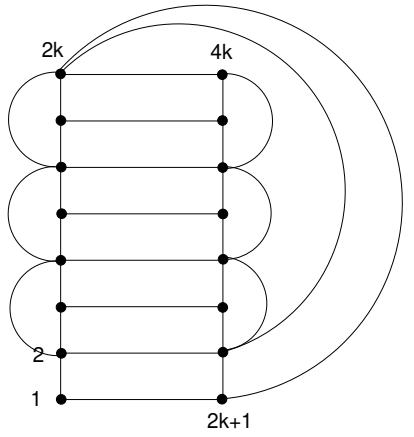

(b) Graph $P_{3}$

Figure 6

edge set $C_{2 k} \cup\left\{d_{2}, d_{3}\right\}$ with $d_{2}, d_{3} \neq d_{1}$. It follows by elementary matrix manipulations that the $3 k$ incidence vectors are all linearly independent. They also satisfy the $V_{2 k}$ inequality with equality. Hence the theorem follows.

Theorem 6 For the graph $F_{4 k}=(V, E)$ with $E=C_{4 k} \cup D_{4 k} \cup B_{4 k}$ the flower inequality

$$
(2 k-3) x\left(B_{4 k}\right)+x\left(C_{4 k}\right)+x\left(D_{4 k}\right) \leq 4 k^{2}-(2 k-2)
$$

is facet-defining for $\mathcal{P} \mathcal{L} S\left(F_{4 k}\right)$.

Proof. The flower inequality says essentially that we have to delete edges with weight at least $2 k-2$ from $F_{4 k}$ in order to get a planar subgraph. Let us assume that there is a planar graph induced by the edge set $P$ whose incidence vector violates the flower inequality. If $C_{4 k} \subseteq P$, all but two edges of $D_{4 k}$ must be deleted in $F_{4 k}$ in order to obtain a planar graph, so the flower inequality is satisfied, which is a contradiction. If at least one edge of $B_{4 k}$ is not contained in $P$, we already removed edges of $F_{4 k}$ with weight $2 k-3$. Since also one edge of $C_{4 k}$ is not contained in $P$, the flower inequality cannot be violated by $\chi^{P}$.

Hence we have $B_{4 k} \subseteq P$. Let us assume that $P$ contains $B_{4 k}$ and exactly $4 k-i$ edges of $C_{4 k}$ for $i \in\{0,1, \ldots, 4 k\}$. Since the flower inequality is violated by $\chi^{P}$ it follows that at most $(2 k-3)-i$ edges of $D_{4 k}$ are not contained in $P$. For $i \geq k$ this implies that at most $k-3$ edges of $D_{4 k}$ are missing in $P$. On the other hand, the edges in $D^{k}:=\{(2,2+2 k),(4,4+2 k), \ldots,(2 k, 4 k)\}$ are pairwise interlacing with respect to cycle $C^{k}:=(2,4, \ldots, 4 k) \subseteq B_{4 k}$, which means that no two edges of $D^{k}$ can be embedded to the same side of $C^{k}$. This implies that at least $\left|D^{k}\right|-2=k-2$ edges of $D_{4 k}$ cannot be contained in $P$, which is a contradiction. Therefore we have $i \in\{0,1, \ldots, k-1\}$. Let $i=k-1$ and define the index set $J^{k}:=\{2,4, \ldots, 4 k\}$. Then there exists a $j \in J^{i+1}$ such that $\{(j, j+1),(j+1, j+2),(j+2 k, j+2 k+1),(j+2 k+1, j+2 k+2)\} \subseteq C_{4 k}$ is contained in $P$. We define $C^{i}:=C^{i+1} \backslash\{(j, j+2),(j+2 k, j+2 k+2)\} \cup\{(j, j+1),(j+1, j+2),(j+2 k, j+2 k+$ $1),(j+2 k+1, j+2 k+2)\}$ and $D^{i}:=D^{i+1} \cup\{(j+1, j+1+2 k)\}$. Now all the edges in $D^{i}$ are pairwise interlacing with respect to cycle $C^{i} \subseteq P$. Thus at least $\left|D^{i}\right|-2=k+(k-i)-2$ edges of $D_{4 k}$ are not contained in $P$. Altogether, at least $i+(2 k-i-2)=2 k-2$ edges of $C_{4 k} \cup D_{4 k}$ are not contained in $P$, which is a contradiction. We define $J^{i}:=J^{i+1} \backslash\{j\}$. The above arguments apply while decreasing the value of $i$ down to 0 . Hence validity is shown.

Let us assume there is a facet-defining inequality $a^{T} x \leq a_{0}$ which dominates the above inequality $c^{T} x \leq c_{0}$. We show that $c_{i}=\lambda a_{i}$ and $c_{0}=\lambda a_{0}$ for $\lambda>0$. Let us consider the planar graph $P_{1}$ given by deleting $2 k-2$ edges of $D_{4 k}$ in $F_{4 k}$. By substituting one of the two remaining edges in $D_{4 k}$ by any of the other $2 k-2$ edges, we again obtain a planar graph. Hence all the coefficients of edges in $D_{4 k}$ are equal. If we delete one edge $e_{c} \in C_{4 k}$ in $P_{1}$, we can get a planar graph $P_{2}$ 
by adding a third edge $e_{d} \in D_{4 k}$ (see Figure 6a). The incidence vectors of $P_{1}$ and $P_{2}$ satisfy the inequality $c^{T} x \leq c_{0}$ and hence $a^{T} x \leq a_{0}$ with equality, so we have $0=\chi^{P_{1}}-\chi^{P_{2}}=a_{e_{c}}-a_{e_{d}}$ and hence $a_{e_{c}}=a_{e_{d}}$. Because of symmetry (delete any edge in $C_{4 k}$ ), we get $a_{e_{c}}=a_{e_{d}}$ for any pair of edges $e_{c} \in C_{4 k}$ and $e_{d} \in D_{4 k}$. The incidence vector of the graph $P_{3}$ obtained from $G_{4 k}$ by removing one edge $(2 i, 2 i+2) \in B_{4 k}$ and one of the set $\{(2 i, 2 i+1),(2 i+1,2 i+2)\} \subseteq C_{4 k}$ is planar and satisfies the equality $c^{T} x=c_{0}$ and hence $a^{T} x=a_{0}$ (see Figure $6 \mathrm{~b}$ for $i=2 k$ ). Thus we get $0=\chi^{P_{1}}-\chi^{P_{3}}=a_{e_{(2 i, 2 i+2)}}+a_{e_{c}}-(2 k-2) a_{e_{d}}$, hence $a_{e_{(2 i, 2 i+2)}}=(2 k-3) a_{e_{d}}$ for $e_{d} \in D_{4 k}$ and $e_{c} \in C_{4 k}$. Because of symmetry we get $a_{e_{b}}=(2 k-3) \lambda, a_{e_{d}}=\lambda, a_{e_{c}}=\lambda$ for $e_{b} \in B_{4 k}, e_{d} \in D_{4 k}$, $e_{c} \in C_{4 k}$ and $c_{0}=\lambda a_{0}$.

\subsection{Operations which yield new facet-defining inequalities}

In the following we will see that some operations like "edge splitting" and "edge contraction" keep the facet-defining property of an inequality. Let $c^{T} x \leq c_{0}$ be an inequality defined in $\mathbf{R}^{E}$ and $f$ be an edge in $E$. We say that the inequality $c^{* T} x^{*} \leq c_{0}^{*}$ defined in $\mathbf{R}^{E^{*}}$ is obtained by splitting the edge $\mathbf{f}$ ( $h$ times) in the following sense. The edge $f=(u, w)$ is replaced by a path $P=\left(u=v_{0}, e_{0}, v_{1}, \ldots, e_{h}, v_{h+1}=w\right)$ and the weights are given by $c_{0}^{*}=c_{0}+h c_{f}, c_{e_{i}}^{*}=c_{f}$ for $0 \leq i \leq h$, and $c_{e}^{*}=c_{e}$ for each $e$ not contained in $P$. We also define the inverse operation, the (edge) contraction of a path $\mathbf{P}$ where we replace the path $P=\left(v_{0}, e_{0}, v_{1}, \ldots, e_{h}, v_{h+1}\right)$ by the edge $f=\left(v_{0}, v_{h+1}\right)$ if $\operatorname{deg}\left(v_{i}\right)=2$ for $1 \leq i \leq h$ and $c_{e_{i}}=c^{\prime}$ for $0 \leq i \leq h$. In this case $c_{0}^{*}=c_{0}-h c^{\prime}$, $c_{f}^{*}=c^{\prime}$ and $c_{e}^{*}=c_{e}$ for each $e$ not contained in $P$.

Note that for every facet-defining inequality $c^{T} x \leq c_{0}$ the weights $c_{e}$ for all the edges on a path $P=\left(v_{0}, e_{0}, v_{1}, \ldots, e_{h}, v_{h+1}\right)$ with $\operatorname{deg}\left(v_{i}\right)=2$ for $1 \leq i \leq h$ are equal, because the removal of one edge on the path destroys exactly the same subdivisions of $K_{5}$ or $K_{3,3}$ as the removal of an arbitrary other edge on $P$ does. This applies also to the edge $f=\left(v_{0}, v_{h+1}\right)$. Hence we have the following lemma.

Lemma 4 Let $c^{T} x \leq c_{0}$ be facet-defining for $\mathcal{P} \mathcal{L S}(G)$. Then the inequality $c^{* T} x^{*} \leq c_{0}^{*}$ obtained from $c^{T} x \leq c_{0}$ by splitting an edge $f$ or contracting a path $P=\left(v_{0}, e_{0}, v_{1}, \ldots, e_{h}, v_{h+1}\right)$ with $\operatorname{deg}\left(v_{i}\right)=2$ for $1 \leq i \leq h$ is facet-defining for $\mathcal{P} \mathcal{L S}\left(G^{\prime}\right)$, where $G^{\prime}$ denotes the graph obtained by the above substitution.

Consider an inequality which is facet-defining for $\mathcal{P} \mathcal{L S}(G)$. By adding an edge to $G$, which is incident to at most one node in $G$, the planarity or nonplanarity of $G$ is not affected. The sequential lifting theorem for independence systems together with the above remark gives us the following lemma. We call the set of edges which have non-zero coefficients in the inequality $c^{T} x \leq c_{0}$ the support of the inequality.

Lemma 5 (Zero Lifting) Let $G=(V, E)$ be a graph, $U \subseteq E$ and $c^{T} x \leq c_{0}$ a facet-inducing inequality for $\mathcal{P} \mathcal{L S}(G[E \backslash U])$. Choose any $e \in U$ which has at most one endnode incident to the support of $c^{T} x \leq c_{0}$. Then $c^{T} x \leq c_{0}$ defines a facet of $\mathcal{P} \mathcal{L} \mathcal{S}(G[E \backslash U \cup\{e\}])$.

Corollary 3 (Euler inequalities) Let $\left(V^{\prime}, F\right)$ be a clique or a complete bipartite subgraph contained in $G$. Then the Euler inequalities $x(F) \leq 3\left|V^{\prime}\right|-6$ or $x(F) \leq 2\left|V^{\prime}\right|-4$, respectively, are facet-defining for $G$.

Let $G_{1}=\left(V_{1}, E_{1}\right)$ and $G_{2}=\left(V_{2}, E_{2}\right)$ be two graphs induced by the facet-defining inequalities $\sum_{e \in E_{1}} c_{e}^{1} x_{e} \leq c^{1}\left(E_{1}\right)-r_{1}$ and $\sum_{e \in E_{2}} c_{e}^{2} x_{e} \leq c^{2}\left(E_{2}\right)-r_{2}$. Let $e_{1}=\left(u_{1}, v_{1}\right)$ be an edge in $E_{1}$ and $e_{2}=\left(u_{2}, v_{2}\right)$ be an edge in $E_{2}$. By identifying $u_{1}$ and $u_{2}$ into $u$ and $v_{1}$ and $v_{2}$ into $v$ and deleting edge $(u, v)$ we obtain the 2 -sum $G=G_{1} \oplus_{e_{2}}^{e_{1}} G_{2}$ of $G_{1}$ and $G_{2}$.

In the following let $E_{1}^{\prime}:=E_{1} \backslash\left\{e_{1}\right\}$ and $E_{2}^{\prime}:=E_{2} \backslash\left\{e_{2}\right\}$. Furthermore for $i=1,2$ let $S_{i}^{\prime}$ denote a minimum planarizing $\left(u_{i}, v_{i}\right)$-separating set in $E_{i}^{\prime}$, which is the set of edges $S_{i}^{\prime}$ with minimum value 


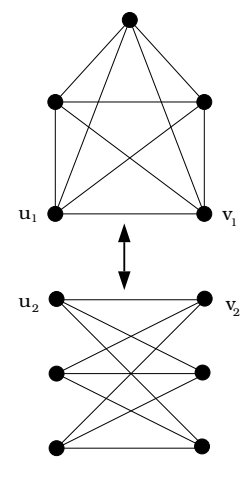

The 2-sum of $\mathrm{K}_{5}$ and $\mathrm{K}_{3,3}$

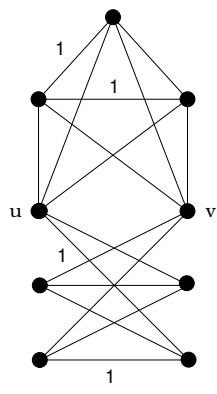

The two support-graphs of the facet-defining inequalities

$c^{i}\left(S_{i}^{\prime}\right)$ whose removal from $E_{i}^{\prime}$ leaves a planar subgraph in which no path between $u_{i}$ and $v_{i}$ exists. The next theorem tells us that we can get two new facet-defining inequalities for $\mathcal{P} \mathcal{L} \mathcal{S}(G)$ by the above described operation provided that some conditions are satisfied.

Theorem 7 For the graphs $G_{1}=\left(V_{1}, E_{1}\right)$ and $G_{2}=\left(V_{2}, E_{2}\right)$ and the edges $e_{1}=\left(u_{1}, v_{1}\right) \in E_{1}$, $e_{2}=\left(u_{2}, v_{2}\right) \in E_{2}$ let $S_{i}^{\prime}$ be a minimum planarizing $\left(u_{i}, v_{i}\right)$-separating set in $E_{i}^{\prime}$. Furthermore, let $s_{i}:=c^{i}\left(S_{i}^{\prime}\right)-r_{i}$ for $i=1,2$. If $c^{1 T} x \leq c^{1}\left(E_{1}\right)-r_{1}$ and $c^{2 T} x \leq c^{2}\left(E_{2}\right)-r_{2}$ are facet-defining inequalities for $\mathcal{P} \mathcal{L S}\left(G_{1}\right)$ and $\mathcal{P} \mathcal{L} \mathcal{S}\left(G_{2}\right)$, respectively, $c_{e_{1}}^{1}>0, c_{e_{2}}^{2}>0$ and the conditions

(a) In $G_{i}$ there exist $\left|E_{i}\right|-1$ different planar subgraphs $P_{j}^{i}$ containing the edge $e_{i}$ whose incidence vectors $\chi^{P_{j}^{i}}$ are linearly independent and satisfy $c^{i T} \chi^{P_{j}^{i}}=c^{i}\left(E_{i}\right)-r_{i}$ for $i=1,2$,

(b) $c_{e_{1}}^{1} c_{e_{2}}^{2} \leq s_{1} s_{2}$,

hold, then $s_{1}>0, s_{2}>0$ and the inequality

$$
c^{T} x \leq c(E)-r
$$

is facet-defining for $\mathcal{P} \mathcal{L} \mathcal{S}\left(G_{1} \oplus_{e_{2}}^{e_{1}} G_{2}\right)$ with

$$
\begin{array}{rlrl}
r & =\lambda_{1} r_{1}+\lambda_{2} r_{2}, & & \\
c_{(k, l)} & =\lambda_{i} c_{(k, l)}^{i} & & \text { for }(k, l) \in E_{i} \text { and } k, l \neq u_{i}, v_{i}, i=1,2, \\
c_{(u, l)} & =\lambda_{i} c_{\left(u_{i}, l\right)}^{i} & & \text { for } l \in V_{i} \backslash\left\{v_{i}\right\}, i=1,2, \\
c_{(k, v)} & =\lambda_{i} c_{\left(k, v_{i}\right)}^{i} & & \text { for } k \in V_{i} \backslash\left\{u_{i}\right\}, i=1,2, \\
\lambda_{1} & =1 \text { and } \lambda_{2} \in\left\{\frac{c_{e_{1}}^{1}}{s_{2}}, \frac{s_{1}}{c_{e_{2}}^{2}}\right\} .
\end{array}
$$

Before we will start to prove the above theorem, we will try to get a feeling about it on the following example. Let $G_{1}=K_{5}$ and $G_{2}=K_{3,3}$. The 2-sum $G_{1} \oplus_{e_{2}}^{e_{1}} G_{2}$ corresponding to the edges $e_{1}=\left(u_{1}, v_{1}\right)$ and $e_{2}=\left(u_{2}, v_{2}\right)$ is shown in Figure 7. $E_{1}^{\prime}=K_{5} \backslash\left\{e_{1}\right\}$ and $E_{2}^{\prime}=K_{3,3} \backslash\left\{e_{2}\right\}$. The value of the minimum planarizing $\left(u_{1}, v_{1}\right)$-separating set in the graph induced by the edge set $E_{1}^{\prime}$ is 3 and the value of the minimum planarizing $\left(u_{2}, v_{2}\right)$-separating set in the graph induced by the edge set $E_{2}^{\prime}$ is 2 . The facet-defining inequality for $G_{1}$ and $G_{2}$ gives us $r_{1}=r_{2}=1$. It follows that $s_{1}=c^{1}\left(S_{1}^{\prime}\right)-r_{1}=3-1=2$ and $s_{2}=c^{2}\left(S_{2}^{\prime}\right)-r_{2}=2-1=1$. So we have $c_{e_{1}}^{1} c_{e_{2}}^{2}=1$, which is less than $s_{1} s_{2}=2$. Hence there are two facet-defining inequalities for $\mathcal{P} \mathcal{L S}\left(G_{1} \oplus_{e_{2}}^{e_{1}} G_{2}\right)$ with $\lambda_{1}=1$ and $\lambda_{2} \in\{1,2\}$.

It is more intuitive to prove the following theorem and then to conclude the above one. 
Theorem 8 For the graphs $G_{1}=\left(V_{1}, E_{1}\right)$ and $G_{2}=\left(V_{2}, E_{2}\right)$ and the edges $e_{1}=\left(u_{1}, v_{1}\right) \in E_{1}$, $e_{2}=\left(u_{2}, v_{2}\right) \in E_{2}$ let $S_{i}^{\prime}$ be a minimum planarizing $\left(u_{i}, v_{i}\right)$-separating set in $E_{i}^{\prime}$ for $i=1,2$. Suppose the inequalities $c^{1 T} x \leq c^{1}\left(E_{1}\right)-r_{1}$ and $c^{2 T} x \leq c^{2}\left(E_{2}\right)-r_{2}$ are facet-defining for $\mathcal{P} \mathcal{L}\left(G_{1}\right)$ and $\mathcal{P L S}\left(G_{2}\right)$, respectively, and the following conditions (a)-(c) hold.

(a) In $G_{i}$ there exist $\left|E_{i}\right|-1$ different planar subgraphs $P_{j}^{i}$ containing the edge $e_{i}$ whose incidence vectors $\chi^{P_{j}^{i}}$ are linearly independent and satisfy $c^{i T} \chi^{P_{j}^{i}}=c^{i}\left(E_{i}\right)-r_{i}$ for $i=1,2$.

(b) There exists a minimum planarizing $\left(u_{i}, v_{i}\right)$-separating set $S_{i}^{\prime}$ in $E_{i}^{\prime}$ for $i=1$ or $i=2$ and $\lambda_{1}, \lambda_{2} \in \mathbf{N}$ with $r=\lambda_{1} r_{1}+\lambda_{2} r_{2}$ such that at least one of the inequalities (i) and (ii) is satisfied with equality and in addition the inequality $c^{i}\left(S_{i}^{\prime}\right) \neq r_{i}$ for the above set $S_{i}^{\prime}$ holds.

(i) $\lambda_{1} c^{1}\left(S_{1}^{\prime}\right) \geq \lambda_{1} r_{1}+\lambda_{2} c_{e_{2}}^{2}$,

(ii) $\lambda_{2} c^{2}\left(S_{2}^{\prime}\right) \geq \lambda_{2} r_{2}+\lambda_{1} c_{e_{1}}^{1}$.

(c) For the specific values of $\lambda_{1}, \lambda_{2}$ satisfying (b) and for the minimum planarizing $\left(u_{i}, v_{i}\right)$ separating sets in $E_{1}^{\prime}$ and $E_{2}^{\prime}$, the inequalities (i) and (ii) hold.

Then inequality (3.1) defined by the 2-sum $G_{1} \oplus_{e_{2}}^{e_{1}} G_{2}$ corresponding to $e_{1}$ and $e_{2}, \lambda_{1}$ and $\lambda_{2}$ is facet-defining for $\mathcal{P} \mathcal{L S}(G)$.

Proof. Let us first show that the new inequality (3.1) is valid. Assume $c^{T} x>c(E)-r$. Then there exists a planar subgraph $P$ in $G_{1} \oplus_{e_{2}}^{e_{1}} G_{2}$ induced by the edge set $P_{1} \cup P_{2}, P_{1} \subseteq E_{1}^{\prime}$ and $P_{2} \subseteq E_{2}^{\prime}$, which yields the inequality $\lambda_{1} c^{1}\left(P_{1}\right)+\lambda_{2} c^{2}\left(P_{2}\right)>\lambda_{1}\left(c^{1}\left(E_{1}^{\prime}\right)-r_{1}\right)+\lambda_{2}\left(c^{2}\left(E_{2}^{\prime}\right)-r_{2}\right)$. Without loss of generality let $c^{1}\left(P_{1}\right)>c^{1}\left(E_{1}^{\prime}\right)-r_{1}$. Suppose now that there is still a $(u, v)$-connecting path in $P_{2}$. In this case the edge set $P_{1}^{\prime \prime}=P_{1} \cup\left\{e_{1}\right\}$ induces a planar subgraph in $E_{1}$. Thus we have $c^{1}\left(P_{1}^{\prime \prime}\right)=c^{1}\left(P_{1}\right)+c_{e_{1}}>\left(c^{1}\left(E_{1}^{\prime}\right)-r_{1}\right)+c_{e_{1}}=c^{1}\left(E_{1}\right)-r_{1}$ which is a contradiction to the validity of the inequality corresponding to $E_{1}$.

Hence $F_{2}:=E_{2}^{\prime} \backslash P_{2}$ is a planarizing $\left(u_{2}, v_{2}\right)$-separating set in $E_{2}^{\prime}$, which implies that $c^{2}\left(F_{2}\right) \geq c^{2}\left(S_{2}\right)$. Moreover, we have

$$
\begin{aligned}
c(P) & =\lambda_{1} c^{1}\left(P_{1}\right)+\lambda_{2} c^{2}\left(P_{2}\right)=\lambda_{1} c^{1}\left(P_{1}\right)+\lambda_{2} c^{2}\left(E_{2}^{\prime}\right)-\lambda_{2} c^{2}\left(F_{2}\right) \\
& \leq \lambda_{1} c^{1}\left(E_{1}\right)-\lambda_{1} r_{1}+\lambda_{2} c^{2}\left(E_{2}^{\prime}\right)-\lambda_{2} c^{2}\left(S_{2}^{\prime}\right) \\
& \leq \lambda_{1} c^{1}\left(E_{1}^{\prime}\right)+\lambda_{1} c_{e_{1}}^{1}-\lambda_{1} r_{1}+\lambda_{2} c^{2}\left(E_{2}^{\prime}\right)-\lambda_{1} c_{e_{1}}^{1}-\lambda_{2} r_{2}=c(E)-r,
\end{aligned}
$$

hence $P$ cannot be a planar subgraph violating inequality (3.1)

In order to prove that inequality (3.1) is facet-defining, we need $\left|E_{1}^{\prime}\right|+\left|E_{2}^{\prime}\right|$ planar subgraphs of $G_{1} \oplus_{e_{2}}^{e_{1}} G_{2}$ whose incidence vectors are linearly independent and satisfy inequality (3.1) as equality. Let $k:=\left|E_{1}^{\prime}\right|$ and $l:=\left|E_{2}^{\prime}\right|$. We take one of the planar subgraphs $P_{j}^{2}$ of $E_{2}$ containing the edge $e_{2}$ whose incidence vector $\chi^{P_{j}^{2}}=\bar{b}_{l}^{T}=\left(b_{l}^{1}, \ldots, b_{l}^{l}, b_{l}^{e_{2}}\right)$ satisfies the equation $c^{2 T} \bar{b}_{l}^{T}=c^{2}\left(E_{2}\right)-r_{2}$ and combine it with each of the planar subgraphs $P_{j}^{1}$ required in (a) for $G_{1}$. The graphs constructed this way are still planar, since both graphs $P_{j}^{i}$ contain the edge $e_{i}$ for $i=1,2$. (Note that the planar graphs $P_{j}^{i}$ can be drawn in a way that the edges $e_{i}$ are adjacent to the outer face). After removing the edges $e_{1}$ and $e_{2}$, we have found $\left|E_{1}^{\prime}\right|$ planar subgraphs whose incidence vectors $\left(\bar{a}_{i}^{T}, \bar{b}_{l}^{T}\right.$ ) (for $i=1, \ldots, k)$ satisfy $c^{T} x=c(E)-r$. We do the same for each of the planar subgraphs of $G_{2}$ containing the edge $e_{2}$. They are combined with one of the subgraphs in $G_{1}$ determined in (a). Their incidence vectors will be denoted as $\left(\bar{a}_{s}^{T}, \bar{b}_{j}^{T}\right)$ for $j=1, \ldots, l-1, s \in\{1, \ldots, k\}$.

We have to prove that the incidence vectors of these $\left|E_{1}^{\prime}\right|+\left|E_{2}^{\prime}\right|-1$ planar subgraphs are linearly independent, which means whenever we have

$$
\mu_{1}\left(\begin{array}{c}
\bar{a}_{1} \\
\bar{b}_{l}
\end{array}\right)+\cdots+\mu_{k}\left(\begin{array}{c}
\bar{a}_{k} \\
\bar{b}_{l}
\end{array}\right)+\nu_{1}\left(\begin{array}{c}
\bar{a}_{s} \\
\bar{b}_{1}
\end{array}\right)+\cdots+\nu_{l-1}\left(\begin{array}{c}
\bar{a}_{s} \\
\bar{b}_{l-1}
\end{array}\right)=0
$$

it follows that $\mu_{1}=\cdots=\mu_{k}=\nu_{1}=\cdots=\nu_{l-1}=0$. 
The requirement of $\left(\mu_{1}+\cdots+\mu_{k}\right) \bar{b}_{l}+\nu_{1} \bar{b}_{1}+\cdots+\nu_{l-1} \bar{b}_{l-1}=0$, where $\bar{b}_{l}$ is exactly the incidence vector of the remaining planar subgraph, gives us $\nu_{1}=\cdots=\nu_{l-1}=0$ and $\mu_{1}+\cdots+\mu_{k}=0$. The remaining equation $\mu_{1} \bar{a}_{1}+\cdots+\mu_{k} \bar{a}_{k}=0$ can only be satisfied if $\mu_{1}=\cdots=\mu_{k}=0$.

We still have to prove that the incidence vector of the planar subgraph defined by the minimum planarizing $\left(u_{i}, v_{i}\right)$-separating set $S_{i}^{\prime}$ determined in (b) satisfies (3.1) with equality and is affinely independent from the above ones. The former is shown by

$$
\begin{aligned}
\lambda_{i} c^{i}\left(P_{i}\right)+\lambda_{j} c^{j}\left(P_{j}\right) & =\lambda_{i} c^{i}\left(E_{i}^{\prime}\right)-\lambda_{i} c^{i}\left(S_{i}^{\prime}\right)+\lambda_{j} c^{j}\left(P_{j}\right) \\
& =\lambda_{i} c^{i}\left(E_{i}^{\prime}\right)-\left(\lambda_{i} r_{i}+\lambda_{j} c_{e_{j}}^{j}\right)+\lambda_{j}\left(c^{j}\left(E_{j}\right)-r_{j}\right) \\
& =\lambda_{i}\left(c^{i}\left(E_{i}^{\prime}\right)-r_{i}\right)+\lambda_{j}\left(c^{j}\left(E_{j}^{\prime}\right)-r_{j},\right.
\end{aligned}
$$

where $i, j \in\{1,2\}$ and $i \neq j$. Without loss of generality let $i=1$. Suppose we can construct its incidence vector $(\bar{n}, \bar{m})$ by the affine combination of the above ones:

$$
\left(\begin{array}{c}
\bar{n} \\
\bar{m}
\end{array}\right)=\mu_{1}\left(\begin{array}{c}
\bar{a}_{1} \\
\bar{b}_{l}
\end{array}\right)+\cdots+\mu_{k}\left(\begin{array}{c}
\bar{a}_{k} \\
\bar{b}_{l}
\end{array}\right)+\nu_{1}\left(\begin{array}{c}
\bar{a}_{s} \\
\bar{b}_{1}
\end{array}\right)+\cdots+\nu_{l-1}\left(\begin{array}{c}
\bar{a}_{s} \\
\bar{b}_{l-1}
\end{array}\right) \text { with } \sum_{i=1}^{k} \mu_{i}+\sum_{j=1}^{l-1} \nu_{j}=1 \text {. }
$$

It follows that there exist $\tau_{1}, \ldots, \tau_{k} \in \mathbf{R}$ with $\tau_{1}+\cdots+\tau_{k}=1$ such that $\bar{n}=\tau_{1} \bar{a}_{1}+\cdots+\tau_{k} \bar{a}_{k}$, since $\bar{a}_{s} \in\left\{\bar{a}_{1}, \ldots, \bar{a}_{k}\right\}$. This implies

$$
c^{1 T} \bar{n}=c^{1 T}\left(\tau_{1} \bar{a}_{1}+\cdots+\tau_{k} \bar{a}_{k}\right)=\tau_{1} c^{1 T} \bar{a}_{1}+\cdots+\tau_{k} c^{1 T} \bar{a}_{k}=\left(\tau_{1}+\cdots+\tau_{k}\right)\left(c^{1}\left(E_{1}^{\prime}\right)-r_{1}\right) .
$$

Hence $c^{1}\left(E_{1}^{\prime}\right)-r_{1}=c^{1 T} \bar{n}=c^{1}\left(E_{1}^{\prime}\right)-c^{1}\left(S_{1}^{\prime}\right)$ which is a contradiction to condition (b).

Proof of Theorem 7. We will show that Theorem 7 is equivalent to Theorem 8 . We will first see that Theorem 7 follows directly from Theorem 8 . The validity of $c^{1 T} x \leq c^{i}\left(E_{i}\right)-r_{i}$ gives us directly $s_{1} \geq 0$ and $s_{2} \geq 0$, since we have to remove an edge set with value greater than $r_{i}$ from $E_{i}$ in order to get a planar graph. By condition (b) of Theorem 7 , together with $c_{e_{1}}^{1}>0$ and $c_{e_{2}}^{2}>0$ we get $s_{1}>0$ and $s_{2}>0$. In order to prove that the conditions (b) and (c) in Theorem 8 are implied by condition (b) in Theorem 7, we show that conditions (b) and (c) in Theorem 8 are satisfied with $\lambda_{1}=1$ and $\lambda_{2} \in\left\{\frac{c_{e_{1}}^{1}}{s_{2}}, \frac{s_{1}}{c_{e_{2}}^{2}}\right\}$ given in Theorem 7. Let us first consider $\lambda_{2}=c_{e_{1}}^{1} / s_{2}$. We have $s_{1} s_{2} \geq c_{e_{1}}^{1} c_{e_{2}}^{2}$ which is equivalent to $\left.c^{1}\left(S_{1}^{\prime}\right)-r_{1}\right)\left(c^{2}\left(S_{2}^{\prime}\right)-r_{2}\right) \geq c_{e_{1}}^{1} c_{e_{2}}^{2}$. We have

$$
\lambda_{1} c^{1}\left(S_{1}^{\prime}\right) \geq \lambda_{1} r_{1}+\left(c_{e_{1}}^{1} / s_{2}\right) c_{e_{2}}^{2}
$$

which is equivalent to (i) of Theorem 8. Furthermore we have

$$
\lambda_{2} c^{2}\left(S_{2}^{\prime}\right)-\lambda_{2} r_{2}=\left(c_{e_{1}}^{1} / s_{2}\right)\left(c^{2}\left(S_{2}^{\prime}\right)-r_{2}\right)=c_{e_{1}}^{1}=\lambda_{1} c_{e_{1}}^{1},
$$

hence (ii) is satisfied with equality. The case for $\lambda_{2}=s_{1} / c_{e_{2}}^{2}$ is completely symmetric. This completes the proof of Theorem 7 .

In order to show equivalence of both Theorems, we will show that the condition (b) in Theorem 7 is implied by the conditions (b) and (c) in Theorem 8. Suppose we have found values for $\lambda_{1}, \lambda_{2}$ and a minimum planarizing $\left(u_{i}, v_{i}\right)$-separating set satisfying all the conditions in Theorem 8 . The inequality in Theorem $8(\mathrm{i})$ can only be satisfied if $c^{1}\left(S_{1}^{\prime}\right) \neq r_{1}$, since otherwise $\lambda_{1}\left(c^{1}\left(S_{1}^{\prime}\right)-r_{1}\right)$ would have value zero. Hence the condition $c_{i}\left(S_{i}^{\prime}\right) \neq r_{i}$ is already implied by (i) and (ii) for $i=1,2$. Let us assume that the inequality (i) in Theorem 8 is satisfied with equality. Then, using the notation $s_{1}=c^{1}\left(S_{1}^{\prime}\right)-r_{1}$, we can compute $\lambda_{2}=\lambda_{1} s_{1} / c_{e_{2}}^{2}$. Since also condition (c) in Theorem 8 is satisfied, we have that the inequality (ii) holds for all planarizing $\left(u_{2}, v_{2}\right)$-separating sets $S_{2}^{\prime}$, hence we have $\lambda_{2} c^{2}\left(S_{2}^{\prime}\right) \geq \lambda_{2} r_{2}+\lambda_{1} c_{e_{1}}^{1}$ which is equivalent to $\lambda_{2} s_{2} \geq \lambda_{1} c_{e_{1}}^{1}$. By substituting the above value for $\lambda_{2}$, we get $s_{1} s_{2} \geq c_{e_{1}}^{1} c_{e_{2}}^{2}$. When assuming that the inequality (ii) in Theorem 8 is satisfied with equality, we get $\lambda_{2}=\lambda_{1} c_{e_{1}}^{1} / s_{2}$ and $s_{1} s_{2} \geq c_{e_{1}}^{1} c_{e_{2}}^{2}$. Hence we have shown that Theorem 8 is equivalent to Theorem 7 .

In the following section we will describe how the above theoretical results can help us to create good separation routines in order to get good upper bounds. 


\section{The algorithm}

We have designed a branch and cut algorithm using facet-defining inequalities for $\mathcal{P} \mathcal{L S}(G)$. The algorithm is similar to the algorithm for the linear ordering problem reported in Grötschel, Jünger and Reinelt [GJR84].

The cutting plane generation as well as the lower bound heuristic is based on a planarity testing algorithm. In order to implement a first version of the branch and cut algorithm, we added only a few lines to an already implemented version of the linear planarity testing algorithm of Hopcroft and Tarjan, which is very fast (see [M92]). Since it is the central part of the algorithm, we will briefly describe it in the following.

The planarity testing algorithm of Hopcroft and Tarjan

At the beginning we call a depth-first-search procedure in order to divide the edge set of the graph $G=(V, E)$ into back edges and tree edges. We start by identifying a cycle $C$. When this cycle is removed from $G$, the graph falls apart into several pieces. The algorithm is called recursively to embed each piece in the plane together with the original cycle. Then the embeddings of the pieces are combined, if possible, to give an embedding of the entire graph.

One may think of successively adding paths consisting of tree edges and one back edge at the end to a previously obtained partial embedding. For more details, see [M92] or [HT74]. In the following we describe some details of the branch and cut algorithm.

\section{Cutting plane generation}

The trivial inequalities are handled implicitly by the LP-solver via lower and upper bounds. At the beginning we also add the inequality $x(E) \leq 3|V|-6$, if it is violated, resp. $x(E) \leq 2|V|-4$ in case $G$ is bipartite, if it is violated.

Let $x$ be an LP-solution produced in the cutting plane procedure applied in some node of the enumeration tree. For $0 \leq \varepsilon \leq 1$ we define $E_{\varepsilon}=\left\{e \in E \mid x_{e} \geq 1-\varepsilon\right\}$ and consider $G_{\varepsilon}=\left(V, E_{\varepsilon}\right)$. For the unweighted graph $G_{\varepsilon}$ the linear planarity testing algorithm of Hopcroft and Tarjan is called. The algorithm stops if it finds an edge set $F$ which is not planar. In case the inequality $x(F) \leq|F|-1$ is violated, we add the inequality to the constraints of the current LP. We remove the back edge of the path, which proved the nonplanarity of $F$ after it was added and proceed with the planarity testing algorithm.

This way we usually find several forbidden subgraphs of the graph $G_{\varepsilon}$ in one run of the planarity testing algorithm. Of course, these forbidden subgraphs do not necessarily define facets of the $\mathcal{P} \mathcal{L} \mathcal{S}$ polytope. However, these subgraphs must contain subgraphs which define facets (see Theorem 2). We try to reduce them to facet-defining inequalities in the following way. Once an edge set $F$ is found, where the inequality $x(F) \leq|F|-1$ is violated, we successively delete one edge $f \in F$ from it, and start again the planarity testing algorithm. If $F \backslash\{f\}$ is planar, we add it again to $F$. In either case we choose a different edge $f \in F$. In at most $|F|$ steps we have reduced $F$ to a set of edges, which induces a minimal nonplanar subgraph. So we have found an inequality $x(F) \leq|F|-1$ which is facet-defining for $\mathcal{P} \mathcal{L S}(G)$ and still violated by the current $\mathcal{L P}$-solution.

We also use a simple heuristic which searches for violated Euler inequalities.

\section{Lower bound heuristic}

After an LP has been solved, we try to exploit the solution to produce a feasible solution. Again, we apply the planarity testing algorithm. This way we produce lower bounds which are useful not only for fathoming nodes in the branch and cut tree but also for fixing variables due to their reduced costs during a cutting plane phase.

After discovering a forbidden substructure, the back edge of the last added path is removed, so that the remaining substructure becomes planar. Since different depth-first-search trees yield different paths and thus different lower bounds, in every call of the planarity testing algorithm the depthfirst-search tree is changed. 
We also implemented a simple random heuristic, where the edges are subsequently added to the graph, if they don't destroy planarity. Our experimental results confirm the results of Cimikowski, who reported that simple random heuristics lead to better results on random graphs than the above described method [C92].

It would be much better to use more powerful heuristics, because in a branch and cut algorithm it is important to get good lower bounds. In a future implementation, we will try the algorithm of Cai, Han and Tarjan [CHT91] and Kant [K92] which yield a maximal planar subgraph. We also plan to try out the deltahedron heuristic of Foulds and Robinson [FR78], the wheel expansion heuristic of Eades et al [EFG82] or simply a greedy heuristic. This should be one of the next steps to improve the quality of the feasible solutions produced in the course of the algorithm.

\section{Branching}

Branching takes place if the current solution is infeasible yet no cutting planes have been found. We choose a variable $x_{e}$ with fractional value as close as possible to $\frac{1}{2}$ and among those one with maximum absolute objective function coefficient.

\section{Computational experiments}

For the implementation of the algorithm we combined the above described adaptions of a previous PASCAL implementation of the planarity testing algorithm [M92] with an adaption of a C-implementation of the branch and cut frame used in Jünger, Reinelt and Thienel [JRT92]. In contrast to the algorithm described there, we neither used sparse graph techniques nor methods for fixing and setting variables by logical implication. Our computational experiments were carried out on a SUN SPARCstation 10 model 20.

We could find only a few papers where computational results are reported. Goldschmidt and Takvorian [GT92] presented some results for triangulated planar graphs of 10, 25, 50 and 100 vertices to which they added incrementally one, two and three edges. Additionally they gave results for two graphs which already occurred in Jayakumar et al. [JTS89] and in Kant [K92]. Another graph occuring in papers about the maximal planar subgraph problem is given by Cimikowski [C92]. In all these cases our algorithm found and proved the optimum solution in a reasonable amount of time (see Table 1 and Table 2). The columns from left to right display the problem name, resp. the origin of the problem, the number of nodes, the number of edges, the value of the best solution found by the author, the value of the best solution found by our algorithm, the quality guarantee

Table 1. Results for the graphs in [GT92]

\begin{tabular}{|c|c|c|c|c|c|c|c|c|c|c|}
\hline Problem & \#Nodes & \#Edges & Sol[GT92] & Solution & Guarantee & BC-nodes & $\# \mathrm{LPs}$ & \#Kurat & \#Euler & Time \\
\hline $\mathrm{g} 10.0$ & 10 & 24 & 24 & 24 & 0.00 & 1 & 1 & 0 & 1 & 0 \\
\hline $\mathrm{g} 10.1$ & 10 & 25 & 24 & 24 & 0.00 & 1 & 1 & 1 & 1 & $\mathbf{0}$ \\
\hline g10.2 & 10 & 26 & 24 & 24 & 0.00 & 1 & 3 & 0 & 1 & $\mathbf{0}$ \\
\hline g10.3 & 10 & 27 & 24 & 24 & 0.00 & 1 & 1 & 0 & 1 & 0 \\
\hline g25.0 & 25 & 69 & 68 & 69 & 0.00 & 1 & 1 & 0 & 1 & 0 \\
\hline g25.1 & 25 & 70 & 69 & 69 & 0.00 & 1 & 2 & 0 & 1 & $\mathbf{0}$ \\
\hline g25.2 & 25 & 71 & 68 & 69 & 0.00 & 1 & 1 & 0 & 1 & $\mathbf{0}$ \\
\hline g25.3 & 25 & 72 & 68 & 69 & 0.00 & 1 & 1 & 0 & 1 & $\mathbf{0}$ \\
\hline g50.0 & 50 & 144 & 129 & 144 & 0.00 & 1 & 1 & 0 & 1 & 1 \\
\hline g50.1 & 50 & 145 & 138 & 144 & 0.00 & 1 & 1 & 0 & 1 & 1 \\
\hline g50.2 & 50 & 146 & 142 & 144 & 0.00 & 1 & 1 & 0 & 1 & 1 \\
\hline g50.3 & 50 & 147 & - & 144 & 0.00 & 1 & 1 & 0 & 1 & 0 \\
\hline $\mathrm{g} 100.0$ & 100 & 294 & 183 & 294 & 0.00 & 1 & 1 & 0 & 1 & 4 \\
\hline g100.1 & 100 & 295 & 215 & 294 & 0.00 & 1 & 1 & 0 & 1 & 4 \\
\hline $\mathrm{g} 100.2$ & 100 & 296 & 234 & 294 & 0.00 & 1 & 3 & 12 & 80 & 13 \\
\hline $\mathrm{g} 100.3$ & 100 & 297 & - & 294 & 0.00 & 1 & 1 & 0 & 1 & 4 \\
\hline
\end{tabular}


Table 2. Graphs from the literature

\begin{tabular}{|c|c|c|c|c|c|c|c|c|c|c|}
\hline Author & \#Nodes & \#Edges & Sol[Auth] & Solution & Guarantee & BC-nodes & \#LPs & \#Kurat & \#Euler & Time \\
\hline [JTS89] & 10 & 22 & 19 & 20 & 0.00 & 1 & 5 & 7 & 1 & 0 \\
\hline [K92] & 45 & 85 & 80 & 82 & 0.00 & 24 & 56 & 83 & 1 & 23 \\
\hline$[\mathrm{C} 92]$ & 60 & 165 & 164 & 164 & 0.00 & $\mathbf{0}$ & 2 & 27 & 1 & 4 \\
\hline [H93b] & 20 & 30 & & 28 & 0.00 & 1 & 8 & 16 & 1 & 1 \\
\hline [H93a] & 34 & 45 & & 43 & 0.00 & 1 & 4 & 5 & 1 & 1 \\
\hline [TBB88] & 43 & 62 & & 58 & 0.00 & 46 & 213 & 257 & 1 & 60 \\
\hline [H93a] & 46 & 64 & & 62 & 0.00 & 1 & 5 & 14 & 1 & 1 \\
\hline$[\mathrm{H93a}]$ & 48 & 69 & & 64 & 0.00 & 258 & 1009 & 705 & 1 & 327 \\
\hline [M93] & 17 & 39 & & 35 & 0.00 & 1 & 2 & 0 & 11 & 0 \\
\hline [M93] & 30 & 56 & & 53 & 0.00 & 8 & 23 & 28 & 1 & 3 \\
\hline [M93] & 45 & 98 & & 88 & 2.20 & 1090 & 3312 & 416 & 144 & 1000 \\
\hline [M93] & 47 & 99 & & 91 & 0.00 & 1234 & 3365 & 406 & 1 & 984 \\
\hline [M93] & 47 & 101 & & 89 & 4.30 & 564 & 2490 & 719 & 130 & 1000 \\
\hline [M93] & 61 & 187 & & 130 & 22.62 & 206 & 1147 & 441 & 1873 & 1000 \\
\hline [FR76] & 8 & 24 & 113 & 113 & 0.00 & 1 & 2 & 0 & 3 & 0 \\
\hline [F92] & 8 & 28 & 1982 & 1982 & 0.00 & 8 & 30 & 15 & 12 & 1 \\
\hline [L92] & 10 & 44 & 1101 & 1105 & 0.00 & 784 & 3454 & 445 & 1830 & 205 \\
\hline
\end{tabular}

Table 3. Triangulated graphs incremented by 10,20,... edges

\begin{tabular}{|c|c|c|c|c|c|c|c|c|c|c|}
\hline Problem & \#Nodes & \#Edges & Sol $[\mathrm{C} 92]$ & Solution & Guarantee & BC-nodes & \#LPs & \#Kurat & \#Euler & Time \\
\hline $\mathrm{g} 100.10$ & 100 & 304 & 264 & 294 & 0.00 & 6 & 58 & 106 & 735 & 252 \\
\hline $\mathrm{g} 100.20$ & 100 & 314 & 294 & 285 & 3.06 & 46 & 234 & 149 & 2063 & 1000 \\
\hline $\mathrm{g} 100.30$ & 100 & 324 & 267 & 272 & 7.48 & 36 & 251 & 111 & 2639 & 1000 \\
\hline $\mathrm{g} 100.40$ & 100 & 334 & 282 & 277 & 5.78 & 22 & 228 & 14 & 3567 & 1000 \\
\hline $\mathrm{g} 100.50$ & 100 & 344 & 294 & 273 & 7.14 & 45 & 208 & 334 & 475 & 1000 \\
\hline $\mathrm{g} 100.60$ & 100 & 354 & 258 & 264 & 9.86 & 36 & 246 & 151 & 1251 & 1000 \\
\hline $\mathrm{g} 100.90$ & 100 & 384 & 261 & 252 & 14.28 & 38 & 241 & 114 & 2246 & 1000 \\
\hline g100.100 & 100 & 394 & 294 & 244 & 17.00 & 30 & 241 & 62 & 2501 & 1000 \\
\hline
\end{tabular}

(((upperbound-lowerbound)/upperbound)*100), the number of branch and cut nodes, the number of LPs, the number of Kuratowski inequalities used by our algorithm, the number of Euler inequalities used by the algorithm and the CPU times in seconds (Fractions of seconds are not shown).

Furthermore, we tested our algorithm on some graphs given by Himsolt [H93a, H93b] in his dissertation about graph drawing. Again, we could solve all of them, although one of them took about 6 minutes. We also tried to solve some problems occuring in VLSI-design given by Martin [M93]. These problems appeared to be very hard to solve. We could not solve all of them to optimality in less than 1000 seconds. The quality guarantee is rather poor on the largest instance.

We also solved some facility layout problems given by Foulds and Robinson [FR76], Foulds [F92] and Leung [L92]. With a heuristic Leung [L92] got a solution value of 1101, whereas we could find and prove the optimum solution value of 1105 in less than 4 minutes (see Table 2).

Cimikowski [C92] considered problem instances in which triangulated planar graphs are augmented by $10,20, \ldots$ edges. We tried our code on such instances (see Table 3 ). Here the limits of our current approach become clear: Only in one case we could find the optimum solution within 1000 seconds of CPU time. Elaborate (and time consuming) heuristics must be added to our implementation in order to make it competitive on harder problem instances.

In order to further explore the limits of our branch and cut algorithm, we tested it on a series of randomly generated graphs. At first we increased the density on graphs with 10 and 20 nodes. We did this for unweighted graphs (see Table 4) and for weighted graphs (see Table 5), where the weights were normally distributed with mean 100 and standard deviation $\sigma=20$. We tried different random seeds, but the variance was not high so that the table gives the right impression. 
Table 4. Increasing the density for unweighted graphs on 10 and 20 nodes

\begin{tabular}{|r|r|r|r|r|r|r|r|}
\hline \#Nodes & \#Density & \#Edges & Solution & Guarantee & BC-nodes & \#LPs & Time \\
\hline 10 & 10 & 4 & 4 & 0.00 & 1 & 1 & 0 \\
10 & 20 & 9 & 9 & 0.00 & 1 & 1 & 0 \\
10 & 30 & 13 & 13 & 0.00 & 1 & 1 & 0 \\
10 & 40 & 18 & 17 & 0.00 & 1 & 2 & 0 \\
10 & 50 & 22 & 20 & 0.00 & 1 & 6 & 0 \\
10 & 60 & 27 & 24 & 0.00 & 1 & 3 & 0 \\
10 & 70 & 31 & 24 & 0.00 & 36 & 273 & 15 \\
10 & 80 & 36 & 24 & 0.00 & 1 & 3 & 0 \\
10 & 90 & 40 & 24 & 0.00 & 1 & 1 & 0 \\
10 & 100 & 45 & 24 & 0.00 & 1 & 1 & 0 \\
\hline 20 & 10 & 19 & 19 & 0.00 & 1 & 1 & 0 \\
20 & 20 & 38 & 36 & 0.00 & 16 & 176 & 16 \\
20 & 30 & 57 & 43 & 10.00 & 86 & 1755 & $\mathbf{3 0 0}$ \\
20 & 40 & 76 & 47 & 7.42 & 122 & 1284 & 300 \\
20 & 50 & 95 & 52 & 3.71 & 112 & 1151 & 300 \\
20 & 60 & 114 & 49 & 0.00 & 12 & 138 & 40 \\
20 & 70 & 133 & 49 & 0.00 & 1 & 8 & 2 \\
20 & 80 & 152 & 53 & 0.00 & 1 & 4 & 1 \\
20 & 90 & 171 & 54 & 0.00 & 1 & 1 & 0 \\
20 & 100 & 190 & 54 & 0.00 & 1 & 1 & 0 \\
\hline
\end{tabular}

Table 5. Increasing the density for weighted graphs on 10 and 20 nodes

\begin{tabular}{|r|r|r|r|r|r|r|}
\hline \#Nodes & \#Density & \#Edges & Guarantee & BC-nodes & \#LPs & Time \\
\hline 10 & 10 & 5 & 0.00 & 1 & 1 & 0 \\
10 & 20 & 9 & 0.00 & 1 & 1 & 0 \\
10 & 30 & 13 & 0.00 & 1 & 1 & 0 \\
10 & 40 & 18 & 0.00 & 1 & 2 & 0 \\
10 & 50 & 22 & 0.00 & 1 & 10 & 0 \\
10 & 60 & 27 & 0.00 & 14 & 157 & 5 \\
10 & 70 & 31 & 0.00 & 258 & 1953 & 79 \\
10 & 80 & 36 & 0.00 & 4 & 20 & 0 \\
10 & 90 & 40 & 0.00 & 192 & 1389 & 68 \\
10 & 100 & 45 & 0.00 & 206 & 1460 & 69 \\
\hline 20 & 10 & 19 & 0.00 & 1 & 1 & 0 \\
20 & 20 & 38 & 0.00 & 58 & 651 & 44 \\
20 & 30 & 57 & 10.03 & 160 & 2573 & 300 \\
20 & 40 & 76 & 9.71 & 158 & 2034 & 300 \\
20 & 50 & 95 & 7.57 & 102 & 1755 & 300 \\
20 & 60 & 114 & 7.61 & 86 & 1531 & 300 \\
20 & 70 & 133 & 2.93 & 88 & 1298 & 300 \\
20 & 80 & 152 & 5.08 & 88 & 1132 & 300 \\
20 & 90 & 171 & 4.82 & 44 & 1018 & 300 \\
20 & 100 & 190 & 5.38 & 42 & 893 & 300 \\
\hline
\end{tabular}

In all cases we stopped the computation after 300 seconds of CPU-time. Experience shows that the results do not improve much with a longer computation time. One can observe that the easiest problem instances are those on sparse graphs and very dense graphs. For weighted graphs the behaviour of our branch and cut algorithm is much worse than for unweighted graphs. We believe that this is due to the fact that we have not yet implemented any heuristic for lower bounds, which makes use of the edge weights.

Since in automatic graph drawing the graphs are relatively sparse, we ran a series of sparse graphs. We increased the number of nodes and defined the number of edges to be 1.5 times the number of nodes and 2 times $|V|$ (see Table 6) for the unweighted case. Computational studies on randomly generated unweighted graphs showed that our algorithm can solve almost all instances of graphs with at most 40 edges. But also on instances with up to 60 edges, the probability that we can find the optimum solution is very high. 
Table 6. Sparse graphs

\begin{tabular}{|r|r|r|r|r|r|r|}
\hline \#Nodes & \#Edges & Solution & Guarantee & BC-nodes & \#LPs & Time \\
\hline 10 & 15 & 14 & $\mathbf{0 . 0 0}$ & 1 & 2 & $\mathbf{0}$ \\
20 & $\mathbf{3 0}$ & 28 & $\mathbf{0 . 0 0}$ & 1 & 5 & $\mathbf{0}$ \\
$\mathbf{3 0}$ & 45 & 42 & $\mathbf{0 . 0 0}$ & 1 & 12 & 1 \\
$\mathbf{4 0}$ & $\mathbf{6 0}$ & 55 & $\mathbf{0 . 0 0}$ & 100 & 894 & 155 \\
50 & 75 & 68 & 2.82 & $\mathbf{9 8}$ & 1070 & $\mathbf{3 0 0}$ \\
$\mathbf{6 0}$ & $\mathbf{9 0}$ & 79 & $\mathbf{6 . 9 7}$ & 52 & 807 & $\mathbf{3 0 0}$ \\
70 & 105 & 93 & $\mathbf{6 . 0 2}$ & 24 & 571 & $\mathbf{3 0 0}$ \\
80 & 120 & 103 & $\mathbf{9 . 6 4}$ & $\mathbf{6}$ & 414 & $\mathbf{3 0 0}$ \\
$\mathbf{9 0}$ & 135 & 112 & 12.38 & 6 & 327 & $\mathbf{3 0 0}$ \\
100 & 150 & 127 & 11.81 & $\mathbf{6}$ & 255 & $\mathbf{3 0 0}$ \\
\hline 10 & 20 & 19 & $\mathbf{0 . 0 0}$ & 1 & 2 & $\mathbf{0}$ \\
20 & 40 & 37 & $\mathbf{0 . 0 0}$ & 58 & 714 & $\mathbf{0}$ \\
$\mathbf{3 0}$ & $\mathbf{6 0}$ & 52 & $\mathbf{3 . 7 4}$ & 110 & 1377 & $\mathbf{3 0 0}$ \\
40 & 80 & 66 & 10.65 & $\mathbf{3 0}$ & 854 & $\mathbf{3 0 0}$ \\
$\mathbf{5 0}$ & 100 & 79 & 13.84 & 12 & 559 & $\mathbf{3 0 0}$ \\
$\mathbf{6 0}$ & 120 & $\mathbf{9 0}$ & 19.30 & $\mathbf{6}$ & 350 & $\mathbf{3 0 0}$ \\
70 & 140 & 106 & 20.32 & 4 & 249 & $\mathbf{3 0 0}$ \\
80 & 160 & 117 & 21.73 & $\mathbf{6}$ & 189 & $\mathbf{3 0 0}$ \\
$\mathbf{9 0}$ & 180 & 132 & 25.49 & 1 & 134 & $\mathbf{3 0 0}$ \\
100 & 200 & 139 & 26.50 & 4 & 102 & $\mathbf{3 0 0}$ \\
\hline
\end{tabular}

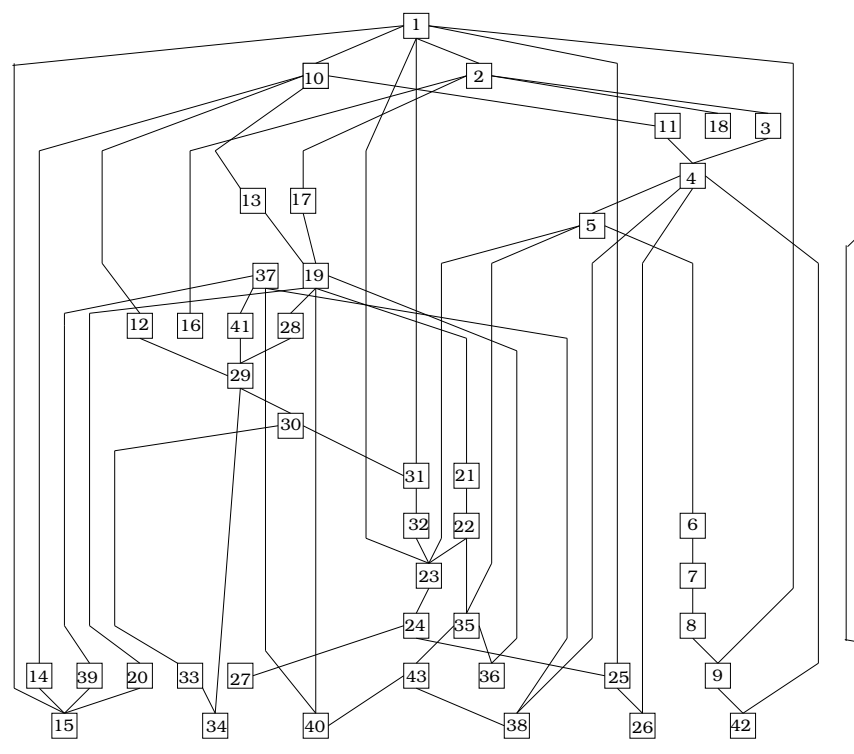

Figure 8(a)

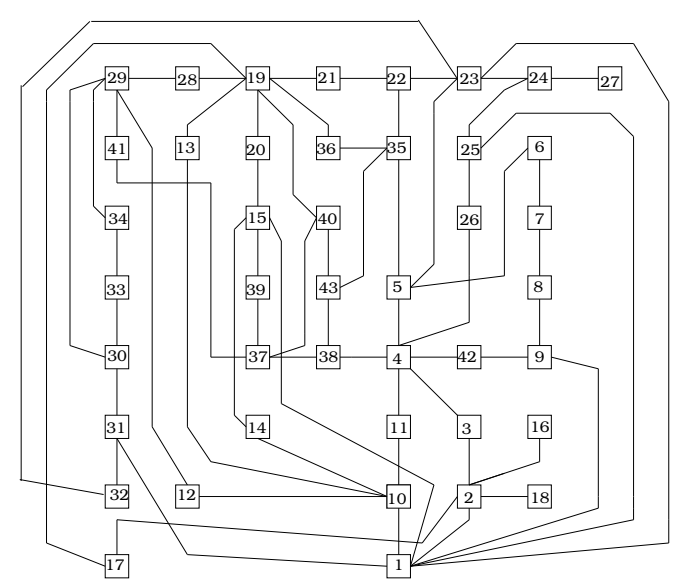

Figure $8(\mathrm{~b})$

Finally, we tested our implementation for a graph given by Tamassia, Di Battista and Batini in a paper about automatic graph drawing [TBB88] (see Figure 8(a)). In order to get the maximum planar subgraph the algorithm removed four of the 62 edges ( 60 seconds). For the embedding of the planar subgraph we used the program of Mutzel [M92]. The insertion of the previously removed edges causes nine crossings, which is much less than the number of crossings in Figure 8(a). The resulting embedding is shown in Figure $8(\mathrm{~b})$.

\section{Final remarks}

Our implementation of a branch and cut algorithm for finding maximum planar subgraphs is very simple in comparison with branch and cut algorithms for other combinatorial optimization problems such as the linear ordering problem [GJR84], or the traveling salesman problem [PR91,JRT92]. If we want to attack bigger problem instances, the most promising refinement of our rather primitive 
implementation is the addition of better separation algorithms, either for facet-defining inequalities which we already know (like the subdivision, $V_{2 k}$, flower or the "composition" inequalities discussed in 2.4) or for new classes of facet-defining inequalities which have yet to be discovered. Another line of attack would be based on preprocessing techniques like scaling or decomposition, and, of course, on implementing more heuristics to improve the lower bounds we get.

Nevertheless, we could solve some problems occuring in the literature to optimality for the first time. This makes us confident that our planned refinements on some of which (and possibly others) we hope to be able to report in a further paper, will lead to a useful algorithm.

\section{Acknowledgements}

We would like to thank two anonymous referees for several helpful remarks, which in particular led to an improvement of the presentation of section 2.4 .

\section{References}

[BL76] Booth, K.S., and G.S. Lueker, "Testing for the consecutive ones property, interval graphs and graph planarity testing using PQ-tree algorithms", J. of Computer and System Sciences 13 (1976) 335-379

[CHT91] Cai, J., X. Han and R.E. Tarjan, "An O $(m \log n)$-Time Algorithm for the Maximal Planar Subgraph Problem", SIAM J. Comput. 22 (1993) $1142-1162$

[C92] Cimikowski, R.J., "An Empirical Analysis of Graph Planarization Heuristics", unpublished manuscript, Computer Science Dept., Montana State Univ. (1992)

[EFG82] Eades P., L.R. Foulds and J.W. Giffin, "An efficient Heuristic for Identifying a Maximum Weight Planar Subgraph", Combinatorial Mathematics IX, Lecture Notes in Mathematics No. 952, Springer-Verlag, Berlin (1982)

[FR76] Foulds, L.R., and R.W. Robinson, "A Strategy for Solving the Plant Layout Problem", Operational Research Quaterly 27 (1976) 845-855

[FR78] Foulds, L.R., and R.W. Robinson, "Graph Theoretic Heuristics for the Plant Layout Problem", Int. J. Production Research 16 (1978) 27-37

[F92] Foulds, L.R., "Graph Theory Applications", Universitext, Springer-Verlag, New York

[GJ79] Garey, M.R., and D.S. Johnson, "Computers and Intractibility: A Guide to the Theory of NP-completeness", Freeman \& Co., San Francisco (1979)

[GT92] Goldschmidt, O., and A. Takvorian, "An efficient graph planarization two-phase heuristic", Technical Report ORP91-01, Dept. of Mech. Engr., Univ. Texas-Austin (1992)

[GJR84] Grötschel, M., M. Jünger, and G. Reinelt, "A cutting plane algorithm for the linear ordering problem", Operations Research 32 (1984) 1195-1220

[H93a] Himsolt, M., "Konzeption und Implementierung von Grapheneditoren", Dissertation, Universität Passau (1993)

[H93b] Himsolt, M., "Personal communication", (1993)

[HT74] Hopcroft, J., and R.E. Tarjan, "Efficient planarity testing", J. ACM 21 (1974) 549-568

[JTS89] Jayakumar, R., K. Thulasiraman, and M.N.S. Swamy, "O $\left(n^{2}\right)$ Algorithms for Graph Planarization", IEEE Trans. on Computer-aided Design 8 (1989) 257-267 
[JRT92] Jünger M., G. Reinelt, and S. Thienel, "Provably good solutions for the traveling salesman problem", Rep. No. 92.114, Angewandte Mathematik und Informatik, Universität zu Köln (1992)

[K92] Kant, G., "An $\mathrm{O}\left(n^{2}\right)$ Maximal Planarization Algorithm based on PQ-trees", Technical Report, RUU-CS-92-03, Dept. of Computer Science, Utrecht University (1992)

[L92] Leung, J., "A new graph-theoretic heuristic for facility layout", Management Science 38 (1992) 594-605

[LG78] Liu, P. C., and R.C. Geldmacher, "On the deletion of nonplanar edges of a graph", Proc. 10th. S-E Conf. on Comb., Graph Theory, and Comp., Boca Raton, FL (1977) $727-738$

[M93] Martin, A., "Personal communication", (1993)

[M92] Mutzel, P., "A fast linear time embedding algorithm based on the Hopcroft-Tarjan planarity test", Rep. No. 92.107, Angewandte Mathematik und Informatik, Universität zu Köln (1992)

[PR91] Padberg, M. W., and G. Rinaldi, "A branch and cut algorithm for the resolution of large-scale symmetric traveling salesman problems", SIAM Review 33 (1991) 60-100

[P89] Pulleyblank, W. R., "Polyhedral combinatorics", in G.L. Nemhauser, A.H.G. Rinnoy Kan and M.J. Todd (eds.), Handbook on Operations Research and Management Sciences: Networks, North Holland 1989

[TBB88] Tamassia, R., G. Di Battista, and C. Batini, "Automatic graph drawing and readability of diagrams", IEEE Transactions on Systems, Man and Cybernetics 18 (1988) 61-79 